\title{
Analytic solution of GPS atmospheric sounding refraction angles
}

\author{
Joseph L. Awange ${ }^{1}$, Yoichi Fukuda ${ }^{1}$, Shuzo Takemoto ${ }^{1}$, Jens Wickert ${ }^{2}$, and Yuichi Aoyama ${ }^{3}$ \\ ${ }^{1}$ Department of Geophysics, Kyoto University, Kitashirakawa Oiwake-cho, Sakyo-ku, Kyoto 606-8502, Japan \\ ${ }^{2}$ GeoForschungsZentrum Potsdam (GFZ), Division 1: Kinematics and Dynamics of the Earth, Telegrafenberg, 14473 Potsdam, Germany \\ ${ }^{3}$ Radio Center for Space and Atmosphere, Kyoto University, Uji, Kyoto 611-0011, Japan
}

(Received December 3, 2003; Revised May 8, 2004; Accepted May 19, 2004)

\begin{abstract}
The nonlinear system of equations for solving GPS atmospheric sounding's bending angles are normally solved using Newton's method. Because of the nonlinear nature of the equations, Newton's method applies linearization and iterations. The method assumes the refraction angle to be small enough such that the dependency of the doppler shift on these angles are linear. The bending angles are then solved iteratively. Since the approach assumes the dependency of doppler shift on bending angles to be linear, which in actual sense is not, some small nonlinearity error is incurred. The Newton's iterative method is often used owing to the bottleneck of solving in exact form the nonlinear system of equations for bending angles. By converting this system of trigonometric nonlinear equations into algebraic, the present contribution proposes an analytic (algebraic) algorithm for solving the bending angles and presents the geometry of the solution space. The algorithm is tested by computing bending angles of three CHAMP occultation data and the results compared to those of iterative Newton's approach. Occultation 133 of 3rd May 2002 is selected as it occurred during diurnal solar radiation maximum past afternoon. During this time, the effect of ionospheric noise is high. Occultations number 3 of 14th May 2001 and number 6 of 2nd February 2002 were selected since they occurred past mid-night, a time of low solar activity and thus less effect of ionospheric noise. The results for occultation 133 of 3rd May 2002 indicate that the nonlinearity errors in bending angles increase with decrease in height to a maximum absolute value of $0.00069^{\circ}(0.1 \%)$ for the region $5-40 \mathrm{~km}$ during period of high solar activity. Such nonlinearity errors are shown to impact significantly on the computed impact parameters to which the bending angles are referred. During low solar activity period, the nonlinearity error was relatively small for occultation number 3 of 14th May 2001 with maximum absolute value of $0.00001^{\circ}$. The analytical algorithm thus provide an independent method for controlling classical iterative procedures and could be used where very accurate results are desired.
\end{abstract}

Key words: Nonlinearity, Sylvester resultant, reduced Groebner basis, Analytical algorithm.

\section{Introduction}

In GPS satellite occultation, the determination of refraction angle $\alpha$ from measured excess phase is the beginning of the computational process to retrieve the atmospheric profiles of temperature, pressure, water vapour and geopotential heights. The trigonometric nonlinear system of equations for solving the bending refraction angles comprise two equations formed by;

1) an equation relating the doppler shift at the LEO expressed as the difference in the projected velocities of the two moving satellites on the ray path tangent on one hand, and the doppler shift expressed as the sum of the atmosphere free propagation term and a term due to atmosphere on the other hand

2) an equation that makes use of Snell's law in a spherically layered medium (Steiner, 1998, p. 59).

Since the equations formed from (1) and (2) are nonlinear, the standard practise has been to apply iterative methods such as Newton's (e.g. Gurbunov et al., 1996; Kursinski et

Copy right (C) The Society of Geomagnetism and Earth, Planetary and Space Science (SGEPSS); The Seismological Society of Japan; The Volcanological Society of Japan The Geodetic Society of Japan; The Japanese Society for Planetary Sciences; TERRAPUB. al., 1997; Steiner, 1998; Wickert, 2002). In order to solve the trigonometric nonlinear system of equations, Newton's approach assumes the refractive angles to be small enough such that the relationship between the doppler shift and the bending angles formed from (1) and (2) are linear. The linearity assumption of the relationship between the doppler shift and refraction angles introduce some small nonlinearity errors. Vorob'ev and Krasil'nikova (1994) have pointed out that the nonlinearity causes an error of $2 \%$ when the beam perigee is close to the Earth's ground and decrease with the altitude of the perigee. The extent of these errors in the dry part of the atmosphere, i.e. the upper troposphere and lower stratosphere, particularly the height 5-30 km, whose bending angle data are directly used to compute the atmospheric profiles or directly assimilated in Numerical Weather Prediction Models (NWPM) (e.g. Healey et al., 2003) is however not precisely stated. The effects of the nonlinearity error on the impact parameters to which the refraction bending angles are related is also not known.

In an attempt to circumvent the nonlinearity problem in the bending angle equations, Vorob'ev and Krasil'nikova (1994) expand the bending angle equations into series of $V / c$ (where $V$ is the velocities of the artificial satellites and $c$ the velocity of light in vacuum) to correct for relativistic effect 


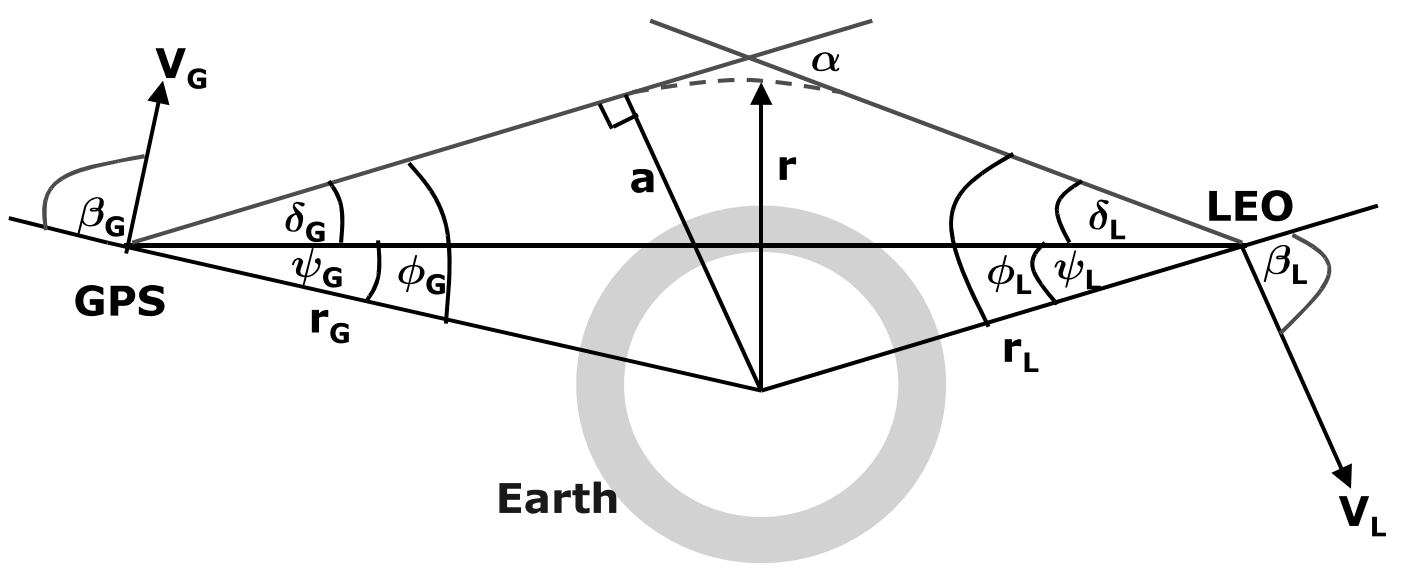

Fig. 1. Geometry of the GPS occultation.

and introduce the concept of perturbation. The angle between the relative position vectors of the two satellites and the tangent velocity vector at GPS is expressed in quadratic terms of the corresponding angle at LEO (also expanded to the second order). The refraction angle is then obtained by making use of infinitesimal refraction angle values that are less than $10^{-2}$. Though the approach attempts to provide an analytic (direct) solution to the nonlinear system of equations for bending angles, it is still never the less "quasi-nonlinear" and as such does not offer a complete exact solution to the problem. Indeed that there existed no direct (exact) solution to nonlinear system of bending angle equations of atmospheric sounding had already been pointed out by Wickert (2002, p. 48).

Motivated by the observation of Wickert (ibid), the present contribution extends on the work of Vorob'ev and Krasil'nikova (1994) by providing an analytic (exact) solution to nonlinear system of equations for bending angles using algebraic approaches of Sylvester resultant (Awange and Grafarend, 2002) and reduced Groebner basis (Awange, 2002; Cox et al., 1998). The developed algorithm is tested by being applied to compute bending angles from the data of CHAMP satellite occultation number 3 of 14th May 2001, number 6 of 2nd February 2002 and number 133 of May 2002. The solutions are then compared to those obtained from iterative procedure in Steiner (1998). Whereas the present contribution focuses on the algorithm, works to be reported in later contribution will consider the effect of nonlinearity error on the computed profiles of density, refractivity, pressure and temperature where a complete nonlinear analysis will be presented. Indeed the analytic (algebraic) algorithm has already been successfully applied in solving nonlinear problems of geodetic nature (e.g. Awange and Grafarend 2003a; Awange et al., 2003 etc.), Robotics for kinematic modelling of robots, Engineering for offset solid modelling, Computer Science for automated theorem proving, and Computer Aided Design.

The present contribution is organized as follows; in Section 2, the nonlinear system of trigonometric equations are converted using Theorem (2-1) of Awange and Grafarend (2003b) into algebraic (polynomial), a necessary condition for applying the algebraic techniques of Sylvester resultant or reduced Groebner basis. Section 3 provides a brief in- troduction to the Sylvester resultant and Groebner basis algebraic approaches which are used to develop an analytic algorithm for solving the nonlinear system of bending angle equations. It is illustrated in Section 4 how the algorithm solve analytically (algebraically) the nonlinear system of bending angle equations once they have been converted into polynomials. In Section 5, the algorithm is tested by being applied to compute the GPS atmospheric sounding bending angles of three given CHAMP satellite occultation data. The contribution is concluded in Section 6.

\section{Conversion of Trigonometric Equations to Alge- braic}

The system of nonlinear trigonometric equations for solving the bending refraction angles comprise two equations given as

$$
\begin{gathered}
v_{L} \cos \left(\beta_{L}-\phi_{L}\right)-v_{G} \cos \left(\phi_{G}+\beta_{G}\right) \\
=\frac{d L_{i}}{d t}+v_{L} \cos \left(\beta_{L}-\psi_{L}\right)-v_{G} \cos \left(\psi_{G}+\beta_{G}\right) \\
r_{G} \sin \phi_{G}=r_{L} \sin \phi_{L},
\end{gathered}
$$

where $v_{L}, v_{G}$ in (1) are the projected LEO and GPS satellite velocities in the occultation plane, $r_{L}, r_{G}$ the radius of tangent points at LEO and GPS respectively and $\frac{d L_{i}}{d t}$, the doppler shift. The angles in (1) are as shown in Fig. 1. For more details, we refer to the works of Steiner (1998) and Wickert (2002). Let us denote

$$
\begin{aligned}
& x=\sin \phi_{G}, y=\sin \phi_{L}, a_{1}=v_{L} \cos \beta_{L}, a_{2}=v_{L} \sin \beta_{L} \\
& a_{3}=-v_{G} \cos \beta_{G}, a_{4}=v_{G} \sin \beta_{G}, a_{5}=r_{G}, a_{6}=-r_{L},
\end{aligned}
$$

where the signs of the velocities in (2) change depending on the directions of the satellites. Using Theorem (2-1) of Awange and Grafarend (2003b), the trigonometric addition formulae and (2), (1) simplifies to

$$
\begin{gathered}
a_{1} \cos \phi_{L}+a_{2} y+a_{3} \cos \phi_{G}+a_{4} x=a \\
a_{5} x+a_{6} y=0 .
\end{gathered}
$$

In (3) the right hand side of the first equation of (1) has been substituted with $a$. In order to eliminate the trigonometric terms $\cos \phi_{L}$ and $\cos \phi_{G}$ appearing in (3), they are taken to the right hand side and the resulting expression squared as

$$
\left(a_{2} y+a_{4} x-a\right)^{2}=\left(-a_{1} \cos \phi_{L}-a_{3} \cos \phi_{G}\right)^{2} .
$$




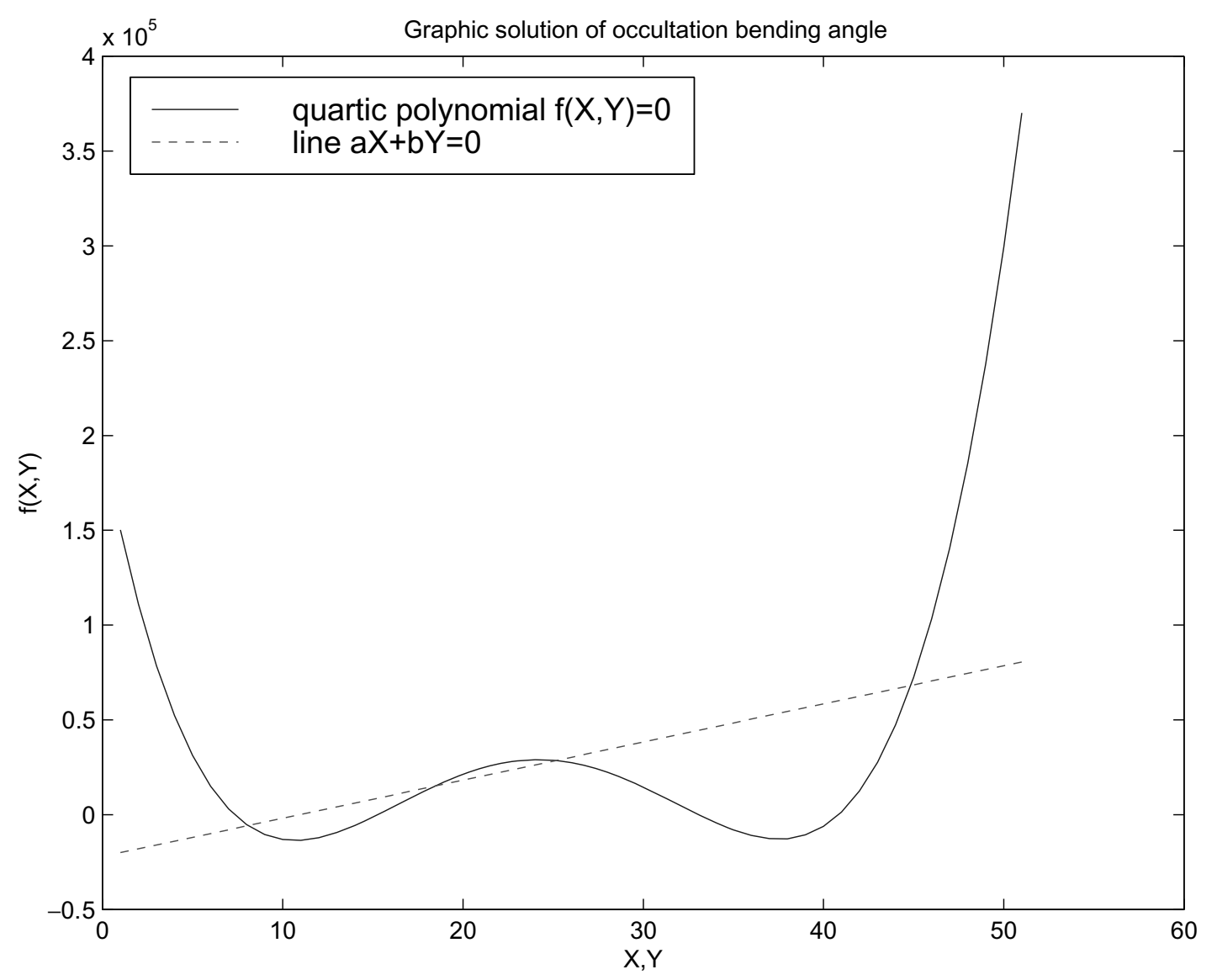

Fig. 2. Algebraic curve for the solution of system of nonlinear GPS atmospheric sounding bending angle equations.

The squared trigonometric values $\cos ^{2} \phi_{G}$ and $\cos ^{2} \phi_{L}$ from (4) are then replaced by variables $x, y$ from (2) using the trigonometric Pythagorean theorem of a unit circle $\cos ^{2} \phi_{G}+$ $\sin ^{2} \phi_{G}=1$ and $\cos ^{2} \phi_{L}+\sin ^{2} \phi_{L}=1$. The resulting expression has only trigonometric product $2 a_{1} a_{3} \cos \phi_{L} \cos \phi_{G}$ on the right hand side. On squaring both sides of the resulting expression and replacing the squared trigonometric values $\cos ^{2} \phi_{G}$ and $\cos ^{2} \phi_{L}$ with $x, y$ from (2) completes the conversion of (1) into algebraic

$$
\begin{gathered}
d_{1} x^{4}+d_{2} x^{3}+d_{3} x^{3} y+d_{4} x^{2}+d_{5} x^{2} y^{2}+d_{6} x^{2} y \\
+d_{7} x+d_{8} x y^{3}+d_{9} x y^{2}+d_{10} x y+d_{00}=0 \\
a_{5} x+a_{6} y=0,
\end{gathered}
$$

with $d_{00}=d_{11} y^{4}+d_{12} y^{3}+d_{13} y^{2}+d_{14} y+d_{15}$. The coefficients $d_{1}, \ldots, d_{15}$ appearing in (5) are as given in the Appendix A. Equation (5) indicates the geometry of the solution space of the nonlinear bending angle equation (1) to be the intersection of a polynomial curve of order four (quartic polynomial) and a straight line. The algebraic curve of (5) is as presented in Fig. 2 indicating that there exist four solutions to the nonlinear problem. Equation (5) can be solved analytically using the Sylvester resultant or Groebner basis algebraic approaches briefly discussed in the next section. The advantage is that both these algebraic approaches are readily available in algebraic software such as Mathematica and Maple.

\section{Algebraic Computing Engine for the Analytic Algorithm}

\subsection{Sylvester resultant approach}

Given two univariate polynomials $p, q \in k[x]$ of positive degree as

$$
\left.\begin{array}{l}
p=k_{0} x^{i}+\cdots+k_{i}, k_{0} \neq 0, i>0 \\
q=l_{0} x^{j}+\cdots+l_{j}, l_{0} \neq 0, j>0
\end{array}\right\}
$$

the resultant of $p$ and $q$, denoted $\operatorname{Res}(p, q)$, is the $(i+j) \times$ $(i+j)$ determinant

$$
\operatorname{Res}(p, q)=\operatorname{det}\left[\begin{array}{cccccccccccc}
k_{0} & k_{1} & k_{2} & . & . & . & k_{i} & 0 & 0 & 0 & 0 & 0 \\
0 & k_{0} & k_{1} & k_{2} & . & . & . & k_{i} & 0 & 0 & 0 & 0 \\
0 & 0 & k_{0} & k_{1} & k_{2} & . & . & . & k_{i} & 0 & 0 & 0 \\
0 & 0 & 0 & k_{0} & k_{1} & k_{2} & . & . & . & k_{i} & 0 & 0 \\
0 & 0 & 0 & 0 & k_{0} & k_{1} & k_{2} & . & . & . & k_{i} & 0 \\
0 & 0 & 0 & 0 & 0 & k_{0} & k_{1} & k_{2} & . & . & . & k_{i} \\
l_{0} & l_{1} & l_{2} & . & . & . & l_{j} & 0 & 0 & 0 & 0 & 0 \\
0 & l_{0} & l_{1} & l_{2} & . & . & . & l_{j} & 0 & 0 & 0 & 0 \\
0 & 0 & l_{0} & l_{1} & l_{2} & . & . & . & l_{j} & 0 & 0 & 0 \\
0 & 0 & 0 & l_{0} & l_{1} & l_{2} & . & . & . & l_{j} & 0 & 0 \\
0 & 0 & 0 & 0 & l_{0} & l_{1} & l_{2} & . & . & . & l_{j} & 0 \\
0 & 0 & 0 & 0 & 0 & l_{0} & l_{1} & l_{2} & . & . & . & l_{j}
\end{array}\right]
$$

In (7), the coefficients of the first polynomial $p$ in (6) occupies $j$ rows while those of the second polynomial $q$ occupies $i$ rows. The empty spaces are occupied by zeros as shown above such that a square matrix is obtained. $k[x]$ indicates the polynomials to have the variable $x$ and the 
coefficients to be in the field $k$ such as $\mathbb{R}$ of real numbers or $\mathbb{C}$ of complex numbers. This resultant is known as the Sylvester resultant and has the following properties (Sturmfels, 1998; Cox et al., 1998, § 3.5).

1) $\operatorname{Res}(p, q)$ is a polynomial in $k_{0}, \ldots, k_{i}, l_{0}, \ldots, l_{j}$ with integer coefficients

2) $\operatorname{Res}(p, q)=0$ if and only if $p(x)$ and $q(x)$ have a common factor in $Q[x] . Q[x]$ is a polynomial ring in the variable $x$ and coefficients in the ring $Q . Q$ can take on any ring such as $\mathbb{R}$ of real numbers or $\mathbb{C}$ of complex numbers etc.

3) There exist a polynomial $r, s \in Q[x]$ such that $r p+$ $s q=\operatorname{Res}(p, q)$

Sylvester resultant can be used to solve a systems of polynomial equations in two variables as shown in Example (3-1).

Example 3-1 Sylvester resultant solution of nonlinear system of equations:

Consider the system of two nonlinear equations

$$
\left.\begin{array}{c}
p:=x y-1=0 \\
q:=x^{2}+y^{2}-4=0
\end{array}\right\}
$$

In order to eliminate one variable e.g. $x$, the variable $y$ is hidden, i.e. the variable say $y$ is considered as a constant (polynomial of degree zero). Sylvester resultant is then computed from (7) as

$\operatorname{Res}(p, q, y)=\operatorname{det}\left[\begin{array}{ccc}y & -1 & 0 \\ 0 & y & -1 \\ 1 & 0 & y^{2}-4\end{array}\right]=y^{4}-4 y^{2}+1$

which can readily be solved for the variable $y$ and substituted back in any of the equations in (8) to get the values of the other variable $x$. Alternatively, the procedure is repeated to solve the variable $x$ directly. Hiding $x$ and applying (7), one obtains

$\operatorname{Res}(p, q, y)=\operatorname{det}\left[\begin{array}{ccc}x & -1 & 0 \\ 0 & x & -1 \\ 1 & 0 & x^{2}-4\end{array}\right]=x^{4}-4 x^{2}+1$.

The roots of the univariate polynomials (9) and (10) are then obtained using Matlab's roots command as

$$
\begin{aligned}
& \{x, y\}=\operatorname{roots}\left(\left[\begin{array}{lllll}
1 & 0 & -4 & 0 & 1
\end{array}\right]\right) \\
& = \pm 1.9319 \text { or } \pm 0.5176 \text {. }
\end{aligned}
$$

In $(11)$, the row vector [ $\left[\begin{array}{lllll}1 & 0 & -4 & 0 & 1\end{array}\right]$ are the coefficients of the quartic polynomials in (9) and (10). The zeros are the coefficients of the variables $\left\{x^{3}, y^{3}\right\}$ and $\{x, y\}$. The solutions in (11) satisfy the polynomials in (9), (10) and the original nonlinear system of equations (8). In equations (9) and (10), the determinant can readily be obtained from MATLAB software by typing $\operatorname{det}(\mathbf{A})$, where $\mathbf{A}$ is the matrix whose determinant is desired.

\subsection{Groebner basis approach}

Groebner basis approach on the other hand reduces a system of multivariate polynomials into a simpler system by using Buchberger algorithm (Buchberger, 1970). Given a system of polynomial equations which are to be solved explicitly for the unknowns, Groebner basis algorithm is applied to reduce this set of polynomials into another set (e.g. from $F(x, y, z)$ to $G(x, y, z))$ of polynomials with suitable properties that allow solution. If $F(x, y, z)$ is a set of nonlinear system of polynomial equations, the application of Groebner basis - which eliminates variables in a manner similar to Gauss elimination technique for linear system of equations-reduces it to another set $G(x, y, z)$ whose solution also satisfy the initial system of nonlinear equations. With lexicographic ordering $x>y>z$ of monomials (e.g. $x$ comes before $y$ which comes before $z$ ), one expression in $G(x, y, z)$ normally turns out to be a univariate polynomial whose roots are easily solvable using algebraic software of Matlab, Mathematica or Maple. Examples (3-2) and (3-3) illustrate the application of Groebner basis.

Example 3-2 Groebner basis solution of nonlinear system of equations:

Let us consider a simple example from Buchberger (2001). Consider a set $F(x, y)=\left\{f_{1}, f_{2}\right\}$ to have as its elements

$$
\begin{array}{r}
f_{1}=x y-2 y \\
f_{2}=2 y^{2}-x^{2}
\end{array}
$$

The Groebner basis algorithm reduces the set in (12) into another set $G$ of $F$ as

$$
G:=\left\{-2 x^{2}+x^{3},-2 y+x y,-x^{2}+2 y^{2}\right\}
$$

In Mathematica software, the Groebner basis could simply be computed by entering the command

$$
\text { Groebner Basis }[\{F\},\{x, y\}]
$$

The set $G$ in (13) contains one univariate polynomial $x^{3}-2 x^{2}$ that can easily be solved e.g. using roots command in Matlab for solutions $(x=0, x=0, x=2)$ and substituted in any of the remaining elements of the set to solve for $y$. If one is interested only in the univariate expression $x^{3}-2 x^{2}$, one computes the reduced Groebner basis in Example (3-3).

Example 3-3 Reduced Groebner basis computation:

In Example (3-2), assuming one desires only those univariate expressions in $x$ and $y$, one computes

$$
\begin{gathered}
\text { Groebner Basis }[\{F\},\{x, y\},\{y\}] \\
\text { Groebner Basis }[\{F\},\{x, y\},\{x\}] .
\end{gathered}
$$

The first expression of (15) will give only $x^{3}-2 x^{2}$ whose roots are already given in Example (3-2). The second expression will give $y^{3}-2 y$ whose roots are $y=0$ or $y= \pm 1.4142$.

For detailed literature on Groebner basis, we refer to standard text books on Groebner bases e.g. Becker and Weispfenning (1998) and Cox et al. (1998). 


\section{Analytic Solution of the Nonlinear Bending An- gle Equations}

\subsection{Solution using Groebner basis}

If the nonlinear system of algebraic (polynomial) equations (5) are denoted as

$$
\begin{gathered}
f_{1}:=d_{1} x^{4}+d_{2} x^{3}+d_{3} x^{3} y+d_{4} x^{2}+d_{5} x^{2} y^{2} \\
+d_{6} x^{2} y+d_{7} x+d_{8} x y^{3}+d_{9} x y^{2}+d_{10} x y+d_{00} \\
f_{2}:=a_{5} x+a_{6} y,
\end{gathered}
$$

using Mathematica software, one solves using reduced Groebner basis (e.g. Example 3-3) for $x$ and $y$ by entering

$$
\begin{gathered}
\text { Groebner Basis }\left[\left\{f_{1}, f_{2}\right\},\{x, y\},\{y\}\right] \\
\text { Groebner Basis }\left[\left\{f_{1}, f_{2}\right\},\{x, y\},\{x\}\right],
\end{gathered}
$$

with the terms $\left\{f_{1}, f_{2}\right\}$ representing the polynomials (16). The second entry in (17) is $\{x, y\}$ which informs Mathematica software of the variables to be solved. The variables are arranged in lexicographic ordering where $x$ comes before $y$. The final entries in (17) are $\{y\}$ and $\{x\}$ which tells Mathematica software which variable to eliminate. The first expression of (17), i.e. Groebner Basis $\left[\left\{f_{1}, f_{2}\right\},\{x, y\},\{y\}\right]$, will give a quartic polynomial in $x$ (i.e. the first expression of (18)), while the second will give a quartic polynomial in $y$ (i.e. the second expression of (18)) as

$$
\begin{aligned}
& h_{4} x^{4}+h_{3} x^{3}+h_{2} x^{2}+h_{1} x+h_{0}=0 \\
& g_{4} y^{4}+g_{3} y^{3}+g_{2} y^{2}+g_{1} y+g_{0}=0,
\end{aligned}
$$

with the coefficients as in Appendix B. Four solutions are obtained from (18) for both $x$ and $y$ using roots command in Matlab software as $x=\operatorname{roots}\left(\left[h_{4} h_{3} h_{2} h_{1} h_{0}\right]\right)$ and $y=\operatorname{roots}\left(\left[\begin{array}{lllll}g_{4} & g_{3} & g_{2} & g_{1} & g_{0}\end{array}\right]\right)$. From (2) and the roots of (18), the required solutions can now be obtained from

$$
\begin{aligned}
& \phi_{G}=\sin ^{-1} x, \\
& \phi_{L}=\sin ^{-1} y .
\end{aligned}
$$

The desired bending angle (see e.g. Fig. 1) is then obtained by first computing $\delta_{G}$ and $\delta_{L}$ as

$$
\begin{gathered}
\delta_{G}=\phi_{G}-\psi_{G}, \\
\delta_{L}=\phi_{L}-\psi_{L}
\end{gathered}
$$

leading to

$$
\begin{gathered}
\alpha=\delta_{G}+\delta_{L}, \\
p=\frac{1}{2}\left(r_{L} \sin \phi_{L}+r_{G} \sin \phi_{G}\right)
\end{gathered}
$$

where $\alpha(p)$ is the bending angle and $p$ the impact parameter.

\subsection{Solution using Sylvester resultant}

The quartic solutions (18) can also be obtained using Sylvester resultant technique as follows:

- Step 1: From the nonlinear system of equation (5), hide $y$ by treating it as a constant (i.e. polynomial of degree zero). From (6) and (7), one computes the resultant of a $5 \times 5$ matrix

$$
\operatorname{Res}\left(f_{1}, f_{2}, y\right)=\operatorname{det}\left[\begin{array}{ccccc}
a_{5} & a_{6} y & 0 & 0 & 0 \\
0 & a_{5} & a_{6} y & 0 & 0 \\
0 & 0 & a_{5} & a_{6} y & 0 \\
0 & 0 & 0 & a_{5} & a_{6} y \\
d_{1} & d_{2}+d_{3} y & b_{53} & b_{54} & b_{55}
\end{array}\right]
$$

with $b_{53}=d_{4}+d_{5} y^{2}+d_{6} y, b_{54}=d_{7}+d_{8} y^{3}+d_{9} y^{2}+$ $d_{10} y$ and $b_{55}=d_{00}$. The solution of (22) leads to the first expression of (18) and can be solved as already described.

- Step 2: From the nonlinear system of equation (5), hide $x$ by treating it as a constant (i.e. polynomial of degree zero). From (6) and (7), one computes the resultant of a $5 \times 5$ matrix

$$
\operatorname{Res}\left(f_{1}, f_{2}, x\right)=\operatorname{det}\left[\begin{array}{ccccc}
a_{6} & a_{5} x & 0 & 0 & 0 \\
0 & a_{6} & a_{5} x & 0 & 0 \\
0 & 0 & a_{6} & a_{5} x & 0 \\
0 & 0 & 0 & a_{6} & a_{5} x \\
d_{11} & d_{12}+d_{8} x & c_{53} & c_{54} & c_{55}
\end{array}\right]
$$

with $c_{53}=d_{13}+d_{5} x^{2}+d_{9} x, c_{54}=d_{14}+d_{3} x^{3}+$ $d_{6} x^{2}+d_{10} x$ and $c_{55}=d_{15}+d_{1} x^{4}+d_{2} x^{3}+d_{4} x^{2}+d_{7} x$. The solution of (23) leads to the second expression of (18) from which the bending angles and the impact parameters can be solved as already discussed.

The analytic algorithm for computing GPS atmospheric sounding refraction angles proceeds in four steps as follows:

Step 1 Coefficient computation:

Using (2) and Appendices A and B, compute the coefficients $\left\{h_{4} h_{3} h_{2} h_{1} h_{0}\right\}$ and $\left\{g_{4} g_{3} g_{2} g_{1} g_{0}\right\}$ of the quartic polynomials (18).

Step 2 Solution for variables $\{x, y\}$ :

Using the coefficients $\left\{h_{i}, g_{i}\right\} \mid i=1,2,3,4$ computed from step 1 , obtain the roots of the univariate polynomials in (18) for $\{x, y\}$.

Step 3 Solve for the angles $\left\{\phi_{G}, \phi_{L}\right\}$ :

With the chosen values of $\{x, y\}$ from step 2 compute using (19) the angles $\left\{\phi_{G}, \phi_{L}\right\}$.

Step 4 Solve for the angles $\left\{\delta_{G}, \delta_{L}\right\}$ :

Using the values of $\left\{\phi_{G}, \phi_{L}\right\}$ from step 3 compute using (20) the angles $\left\{\delta_{G}, \delta_{L}\right\}$.

Step 5 Solve for the angle $\{\alpha\}$ and the impact parameter $p$ : Finally, the bending angle $\{\alpha\}$ and the impact parameter $p$ are computed using the values of $\left\{\phi_{G}, \phi_{L}\right\}$ from step 4 in equation (21).

\section{Test Example}

In order to test the analytic (algebraic) algorithm outlined in steps 1 to 5 to assess the effect of nonlinearity error in using Newton's iterative approach, bending angles from CHAMP satellite level 2 data for three satellite occultations are computed and compared to those obtained from iterative approach in Steiner (1998). The occultations were chosen at different times of the day and years. Occultation number 133 of 3rd May 2002 occurred past mid-day at 13:48:36. For this period of the day, the solar radiation is maximum and so is the ionospheric noise. In contrary, occultation number 3 of 14th May 2001 occurred past mid-night at 00:39:58.00. For this period, the solar radiation is minimum and the effect of ionospheric noise is also minimum. Occultation number 6 of 2nd February 2002 which occurred shortly passed mid-night 


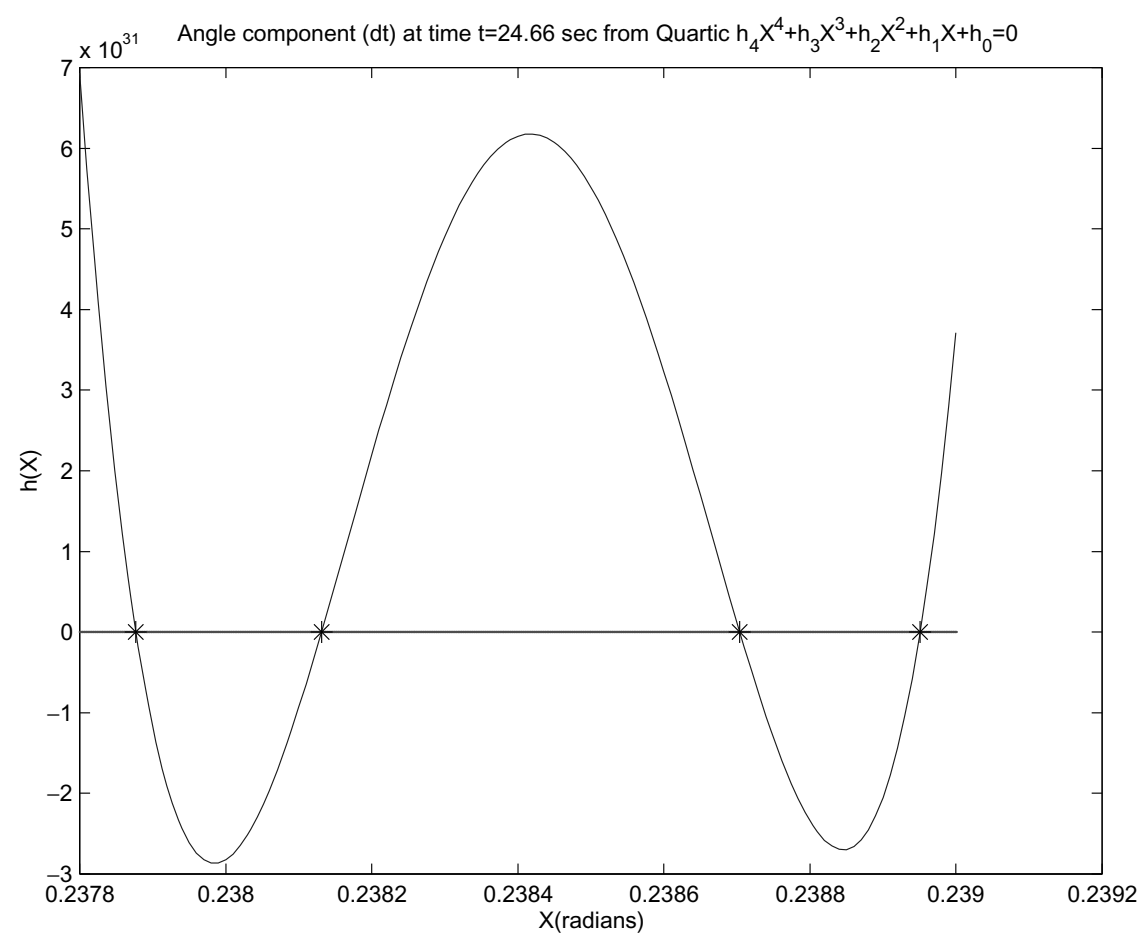

Fig. 3. $x$ values for computing the bending angle component $\delta_{G}$ for $\mathrm{L} 1$ at $t=24.66 \mathrm{sec}$.

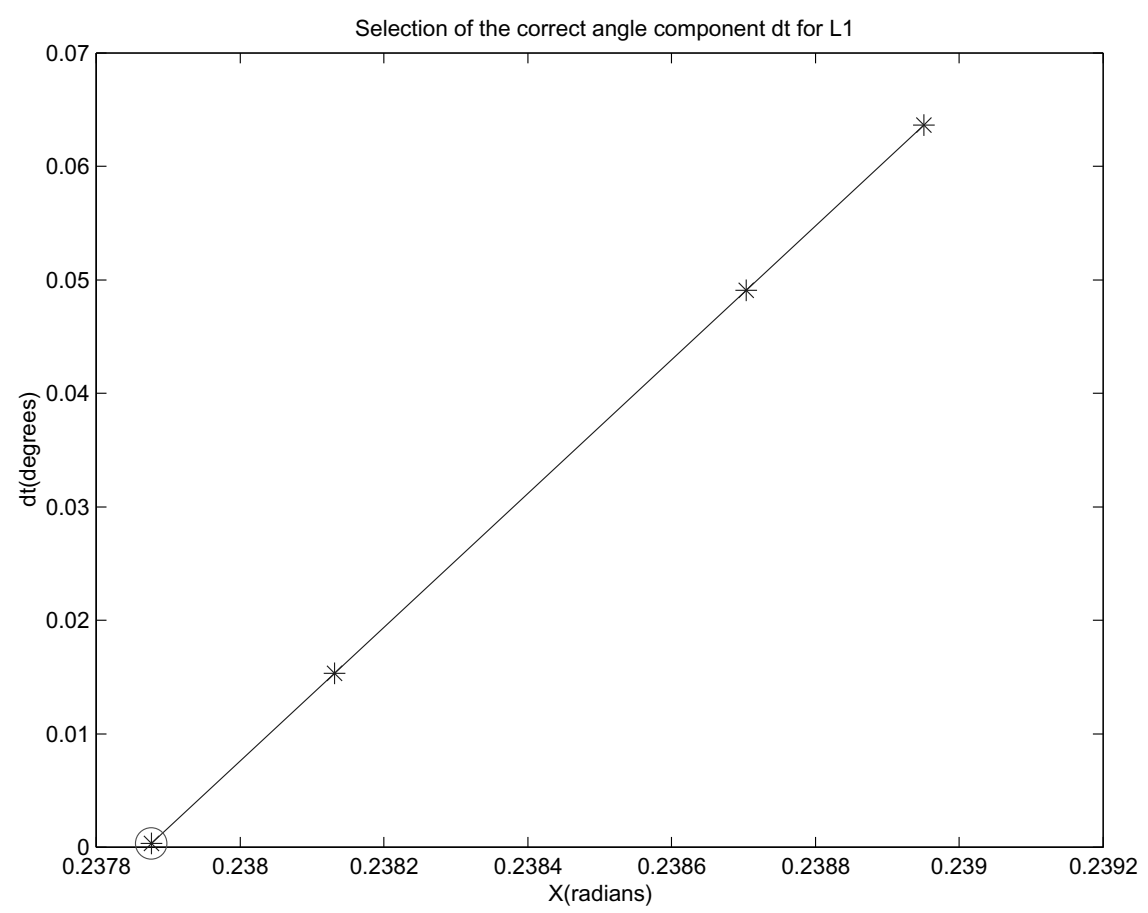

Fig. 4. Selection of the correct $x$ value for computing the component $\delta_{G}$ for L1 at $t=24.66 \mathrm{sec}$.

was further used to test the algorithm.

From the excess phase length data, smoothing was carried out using the polyfit function of Matlab software and the resulting doppler shift values for L1 and L2 used together with (2), (18) and (19) to obtain the angles $\phi_{G}$ and $\phi_{L}$ which were then used in (21) to compute the refraction angle $\alpha$ and the impact parameter $p$ (also denoted in this study as $a$ ). In what follows, a detailed computation of occultation number 133 of 3rd May 2002 during the maximum solar radiation period is presented. The results of occultation number 3 of 14th May 2001 and number 6 of 2nd February 2002 will thereafter be briefly presented. For occultation number 133 of 3rd May 2002 that occurred from the time $13: 48: 36$ to $13: 49: 51.98$, the bending angles were computed using both analytical procedure and the classical Newton's approach presented in Steiner (1998). Since the analytical procedure leads to four solutions as already seen in the preceding sections, a criteria for choosing the correct solu- 


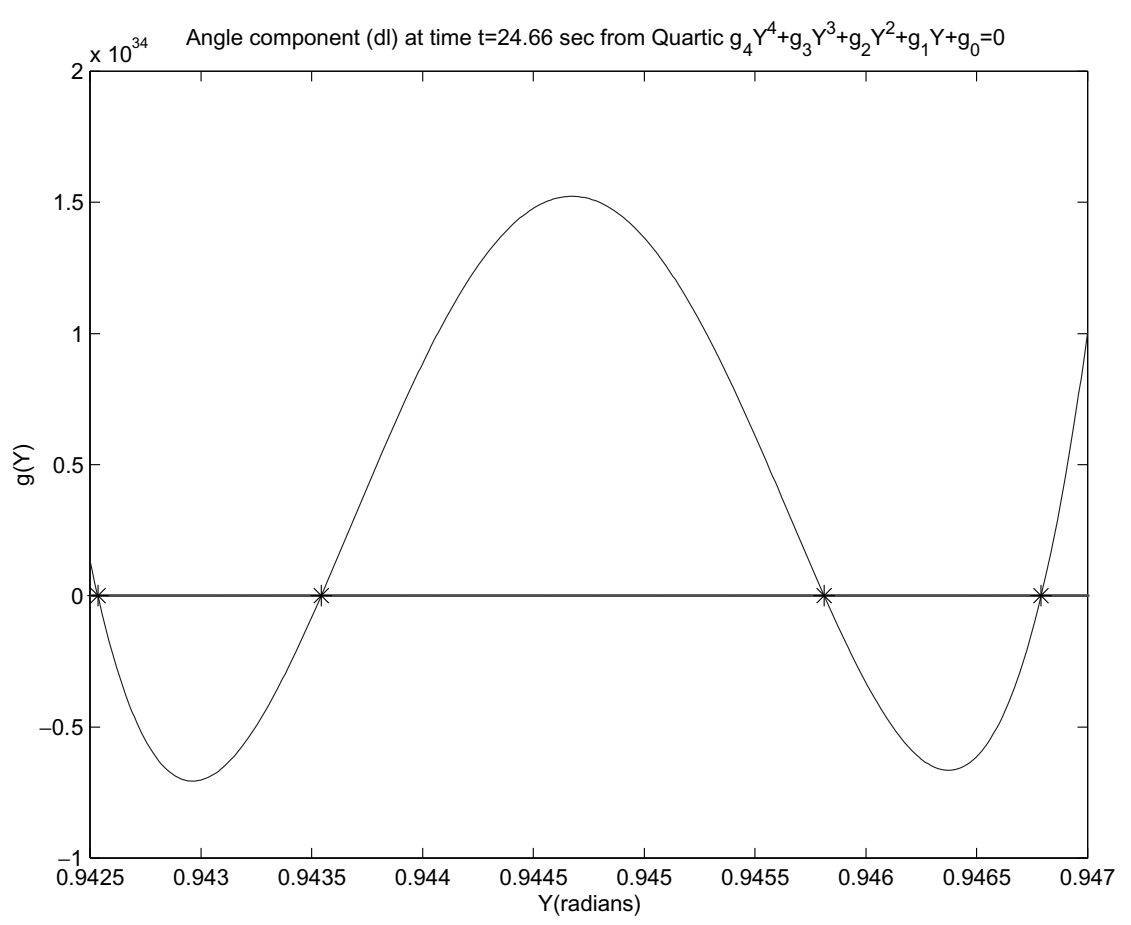

Fig. 5. $y$ values for computing the bending angle component $\delta_{L}$ for L1 at $t=24.66 \mathrm{sec}$.

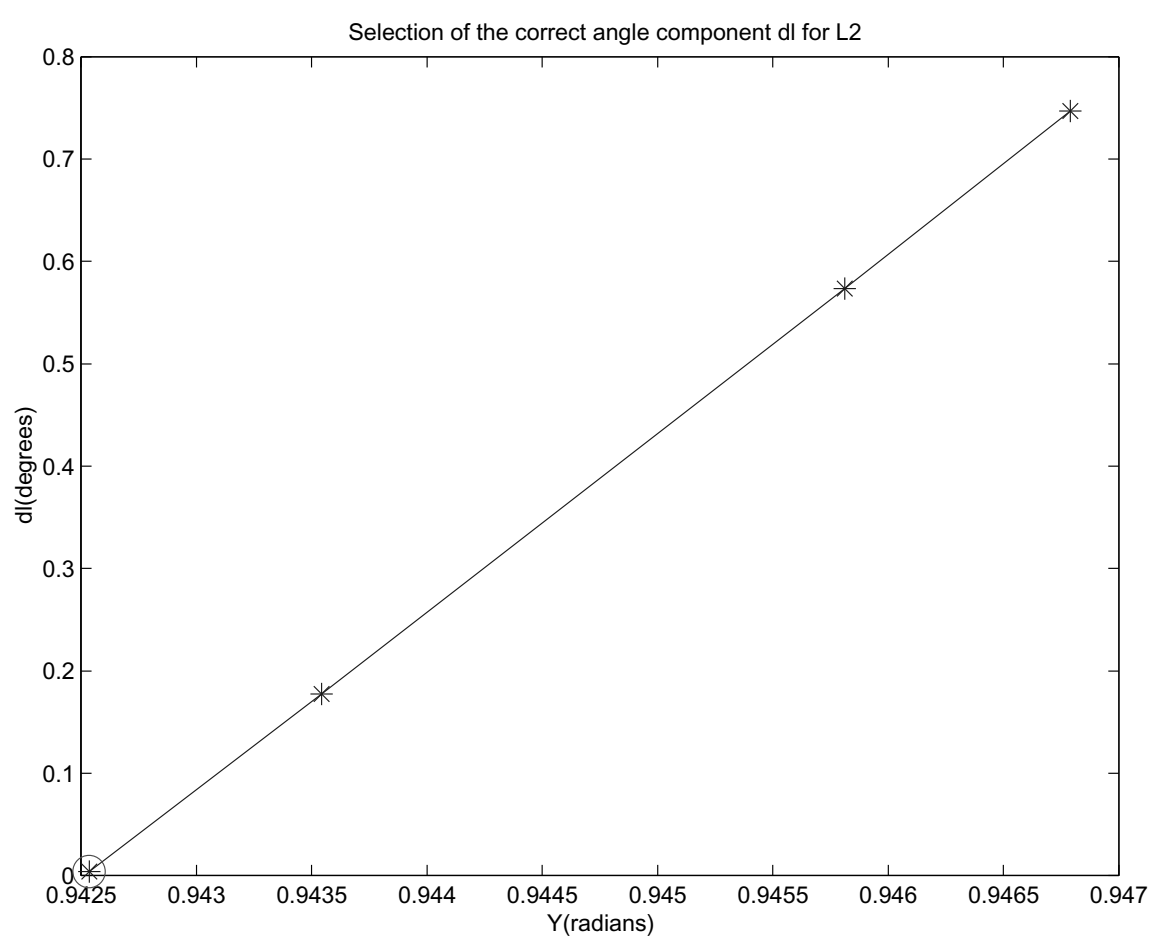

Fig. 6. Selection of the correct $y$ value for computing the component $\delta_{L}$ for L1 at $t=24.66 \mathrm{sec}$.

tion had to be developed. In order to develop this criteria, the bending angles from the classical Newton's approach for time $t=24.66 \mathrm{sec}$ were used as prior information. For this time, solutions from both analytical and classical methods for the L1 signal were compared. Figures 3 and 5 indicate the plot of the four solutions for $x$ and $y$ values computed from equation (18) respectively. These solutions are converted into angular values $\left\{\delta_{G}, \delta_{L}\right\}$ using (19) and (20) respectively and plotted in Figs. 4 and 6. From the values of
Figs. 4 and 6, the smallest values (encircled) were found to be close to those of the classical Newton's solution. In computing the bending angles of the three occultations used in this study, the algorithm was set to select the smallest value amongst the four solutions. Though the Newton's approach converged after three iterations, the iterations in this study were fixed to 20 . The threshold was set such that the difference between the two consecutive solutions were smaller than $1 \times 10 e-06$. 


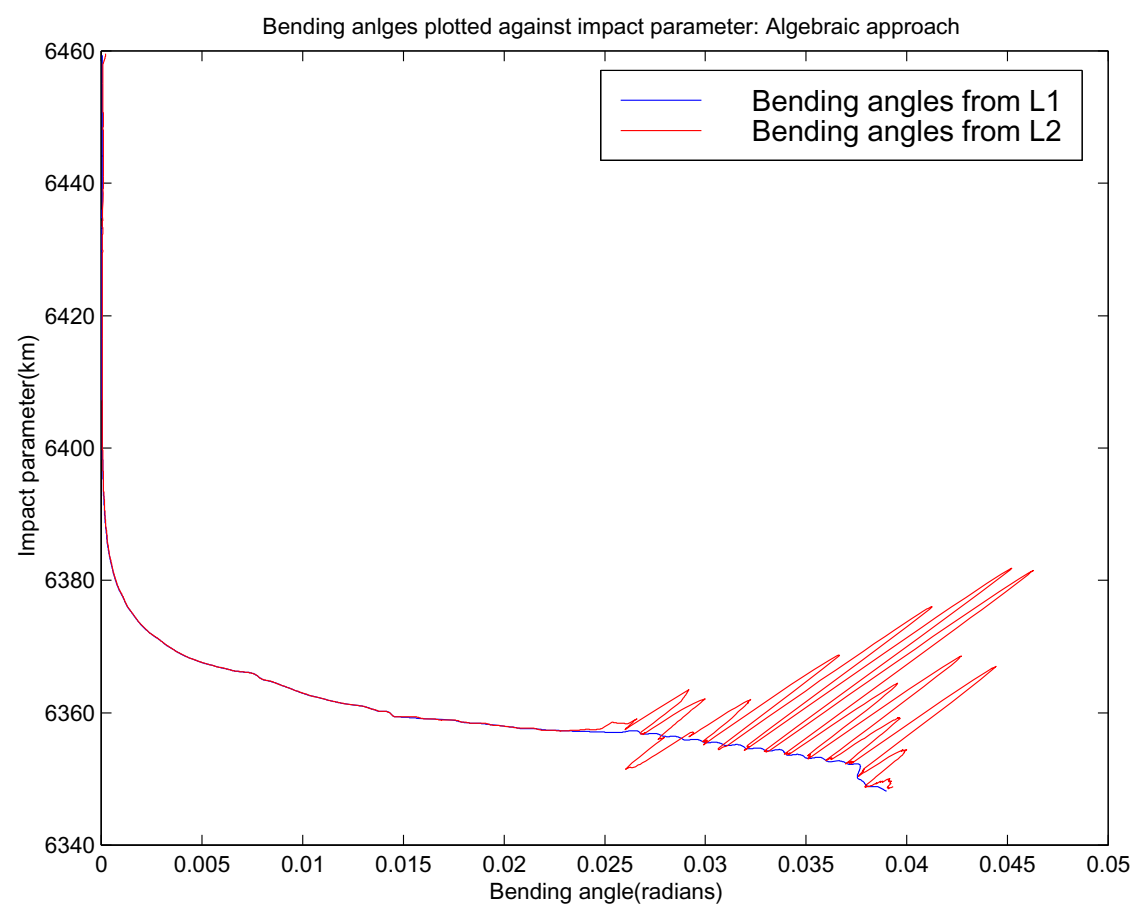

Fig. 7. Bending angles for $\mathrm{L} 1$ and $\mathrm{L} 2$ from analytic algorithm.

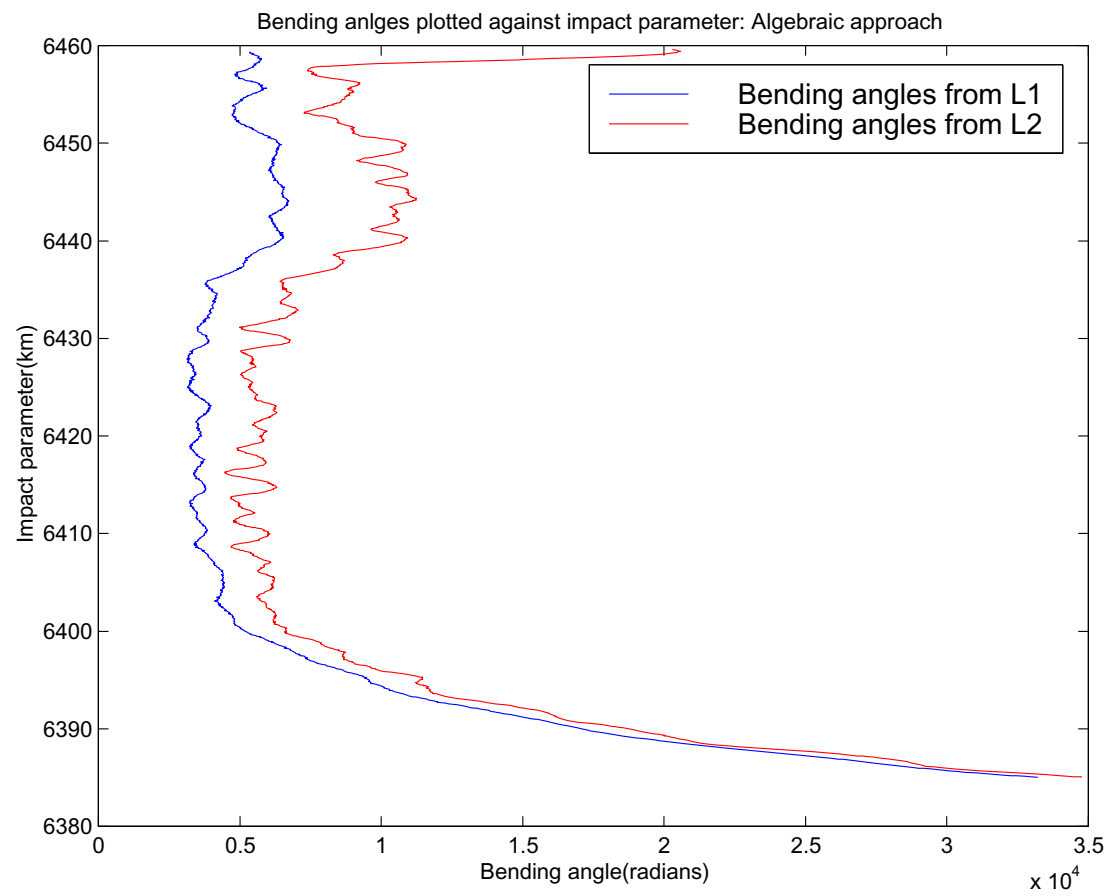

Fig. 8. Magnification of the bending angles above $6380 \mathrm{~km}$ from analytic algorithm.

For the entire occultation, the bending angles $\left\{\alpha=\delta_{G}+\right.$ $\left.\delta_{L}\right\}$ for both L1 and L2 are computed using exact analytic algorithm and are plotted in Fig. 7. A magnification of Fig. 7 above the height $30 \mathrm{~km}$ is plotted in Fig. 8 to show the effect of the residual ionospheric errors on the bending angles from both procedures.

Since bending angle data above $40 \mathrm{~km}$ are augmented with model values and those below $5 \mathrm{~km}$ are highly influenced by the presence of water vapour (see for instance Figs. 7 and 8), the present study restricts its analysis to the bending angles data between 5-40 km. Data in this region are normally used directly to derive the atmospheric profiles required for $\mathrm{Nu}$ merical Weather Prediction models. For occultation number 133 of 3rd May 2002, the differences in both the bending angles $\alpha$ and impact parameters $p$ were computed by subtracting the solutions of the classical Newton's method from those of the analytical approach. The computations were carried out separately for both L1 and L2 signals. In order to compare the results, the computed differences are plotted in Figs. 9, 10, 11 and 12. In these Figures, the vertical axes 


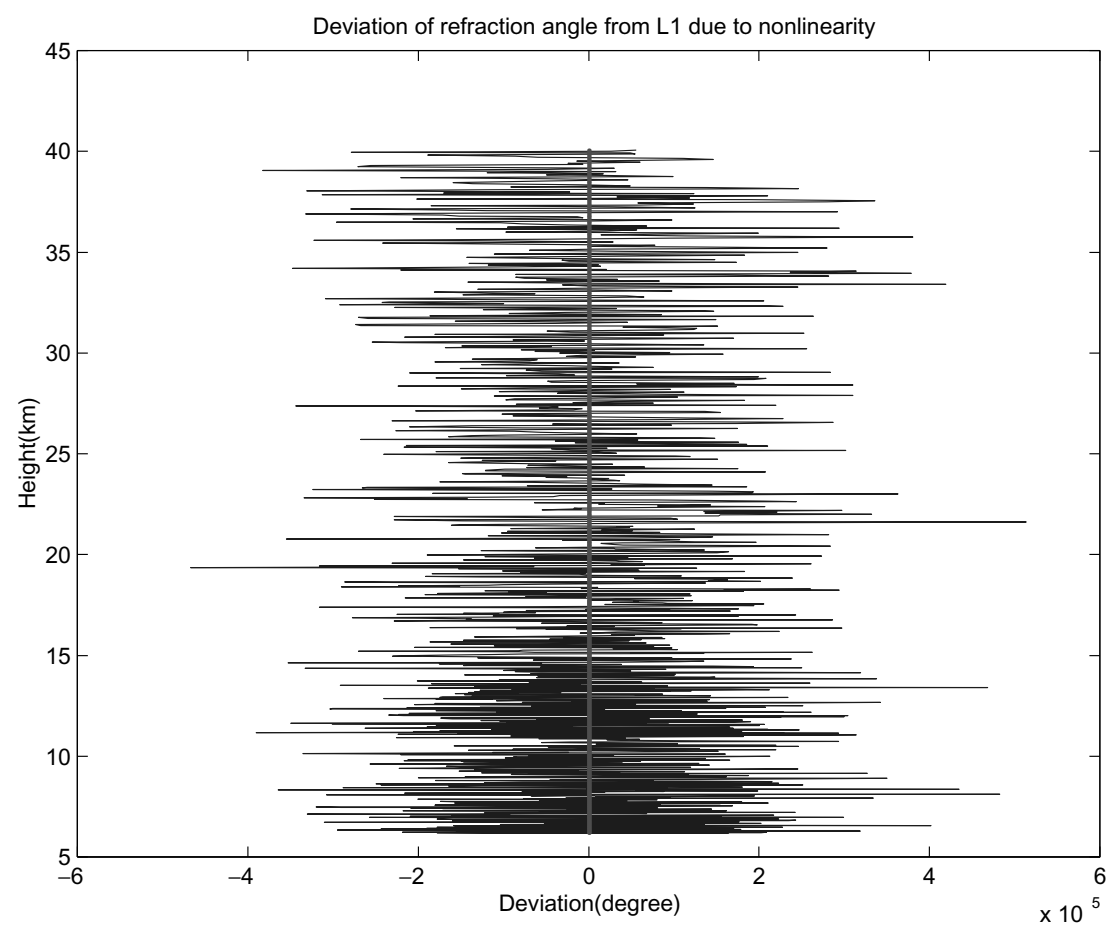

Fig. 9. Differences in computed bending angles from L1 due to nonlinearity for occultation 133 of 3rd May 2002.

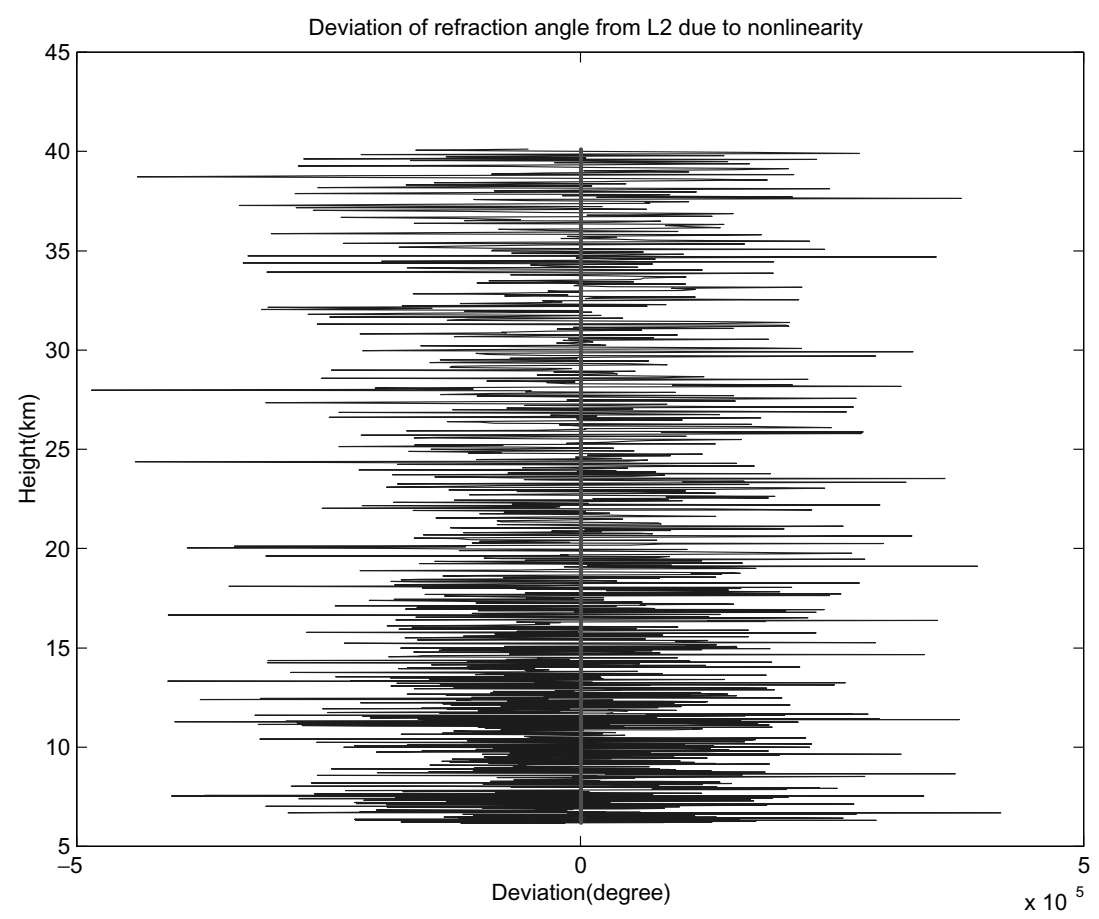

Fig. 10. Differences in computed bending angles from L2 due to nonlinearity for occultation 133 of 3rd May 2002.

are fixed while the horizontal axes indicate the range of the computed differences.

In Figs. 9 and 10, the computed differences in bending angles due to nonlinearity for $\mathrm{L} 1$ are in the range $\pm 6 \times 10^{-5}$ (degrees) with the maximum absolute value of $5.14 \times 10^{-5}$ (degree), while for L2, they are in the range $\pm 5 \times 10^{-5}$ (degrees) with the maximum absolute value of $4.85 \times 10^{-5}$ (degree). The effects of nonlinearity error on the impact parameters for $\mathrm{L} 1$ are in the range $\pm 1.5 \mathrm{~m}$ with the maximum absolute value of $1.444 \mathrm{~m}$, while those of $\mathrm{L} 2$ are in the range $\pm 2 \mathrm{~m}$ with the maximum absolute value of $1.534 \mathrm{~m}$. The large differences in the impact parameters are due to the long distances of the GPS satellites $\left(r_{G}>20,000\right.$ $\mathrm{km}$ ) which are used in the second equation of (21) to compute the impact parameters to which the bending angles are related. Any small difference in the computed bending angles due to nonlinearity therefore contributes significantly to the large differences in the impact parameters. For this par- 


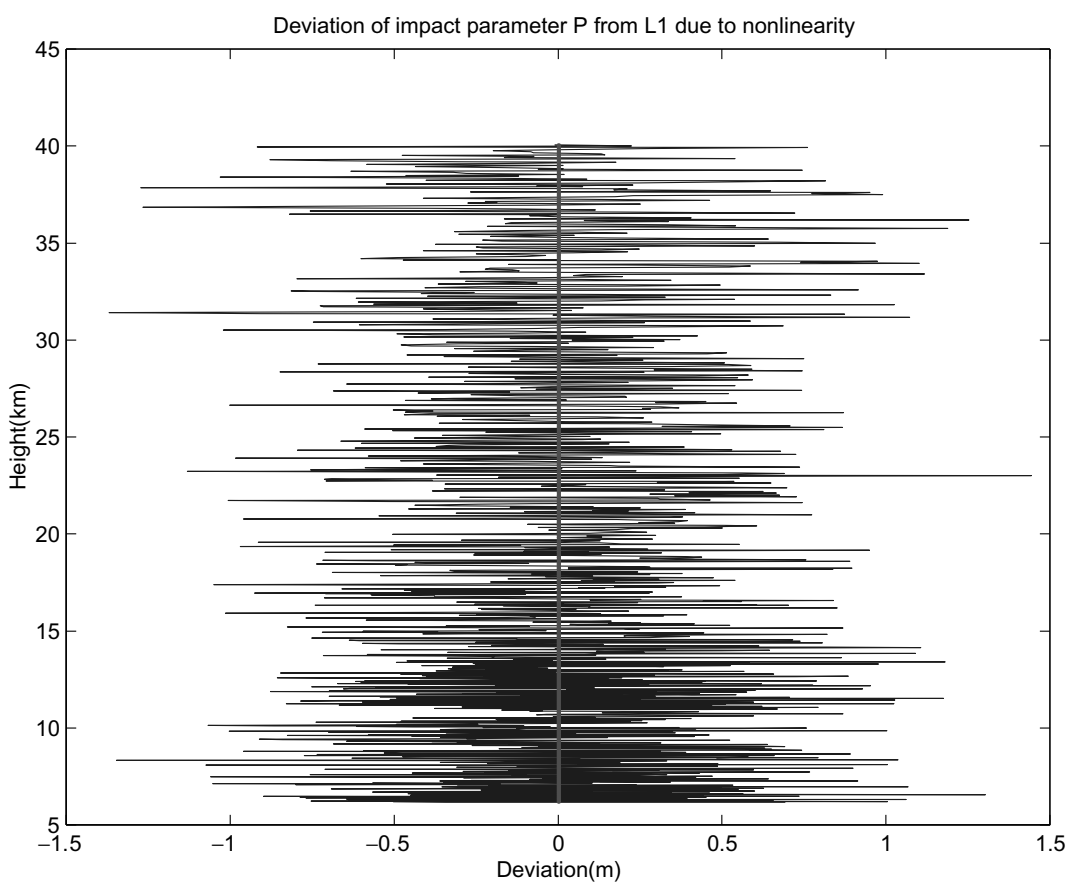

Fig. 11. Differences in computed impact parameters from L1 due to nonlinearity for occultation 133 of 3rd May 2002.

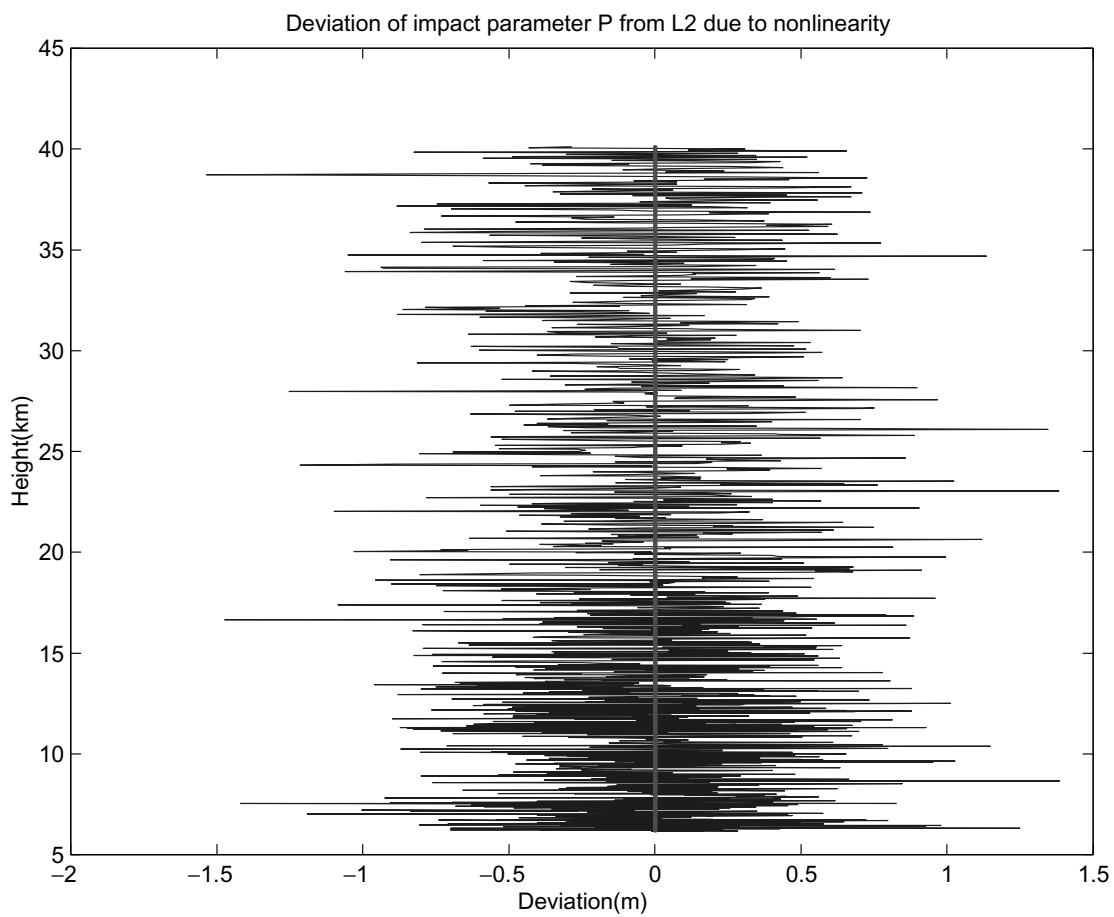

Fig. 12. Differences in computed impact parameters from L2 due to nonlinearity for occultation 133 of 3rd May 2002.

ticular occultation therefore, the bending angles of L1 and L2 signals could probably be related to impact parameters that are off by up to $\pm 2 \mathrm{~m}$.

In order to assess the overall effect of nonlinearity on the bending angles; the bending angles from the analytic (algebraic) algorithm and those of the iterative procedure have to be related to the same impact parameters. In this analysis, the bending angles of L2 from algebraic approach and those of L1 and L2 from iterative approach are all matched through interpolation to the impact parameters $\mathrm{P} 1$ of $\mathrm{L} 1$ from ana- lytic (algebraic) approach. The resulting total bending angles from both algebraic and iterative procedures are then obtained by the linear correction method of Vorob'ev and Krasil'nikova (1994) as

$$
\alpha(a)=\frac{f_{1}^{2} \alpha_{1}(a)-f_{2}^{2} \alpha_{2}(a)}{f_{1}^{2}-f_{2}^{2}} .
$$

The resulting bending angles $\alpha(a)_{i}$ from the iterative approach and $\alpha(a)_{a}$ from analytic (algebraic) approach using (24) are plotted in Fig. 13. The deviation $\nabla \alpha=\alpha(a)_{a}-$ 


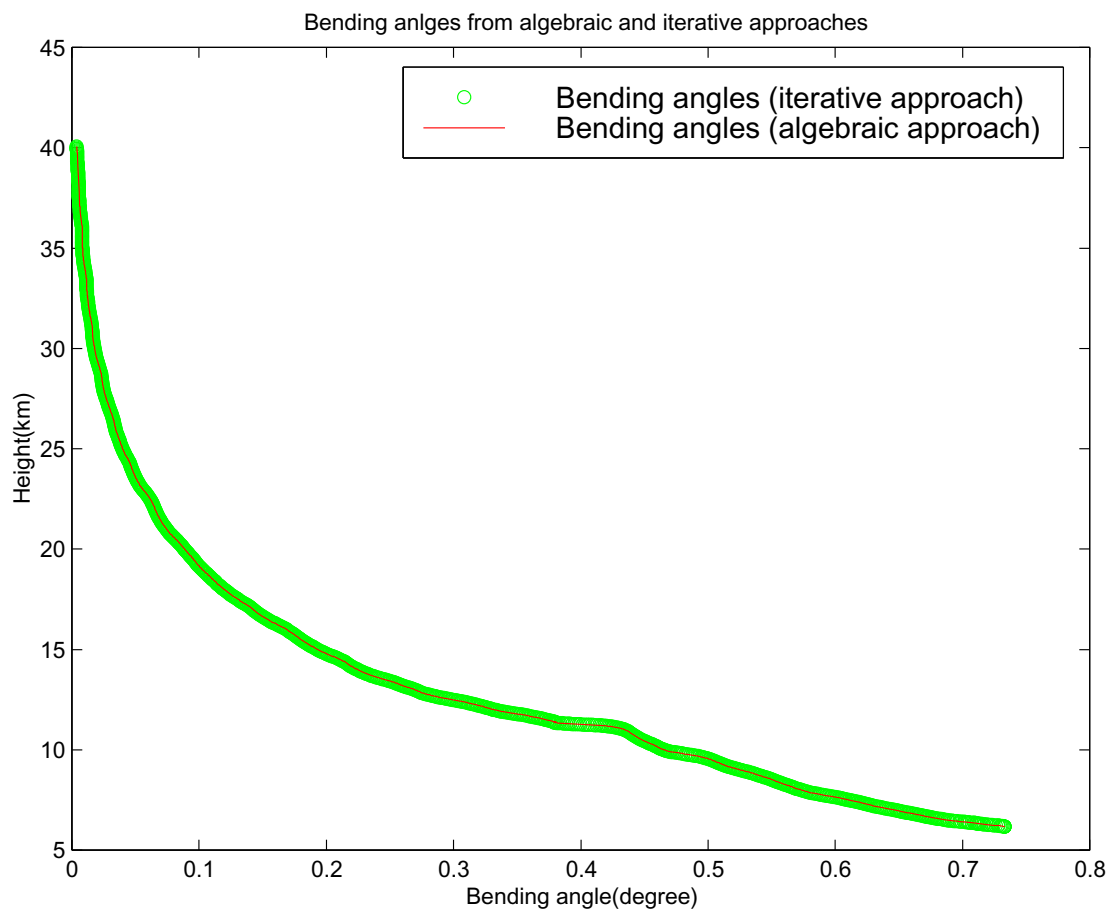

Fig. 13. Bending angles from iterative and analytic algorithms matched to the same impact parameters for occultation 133 of 3rd May 2002.

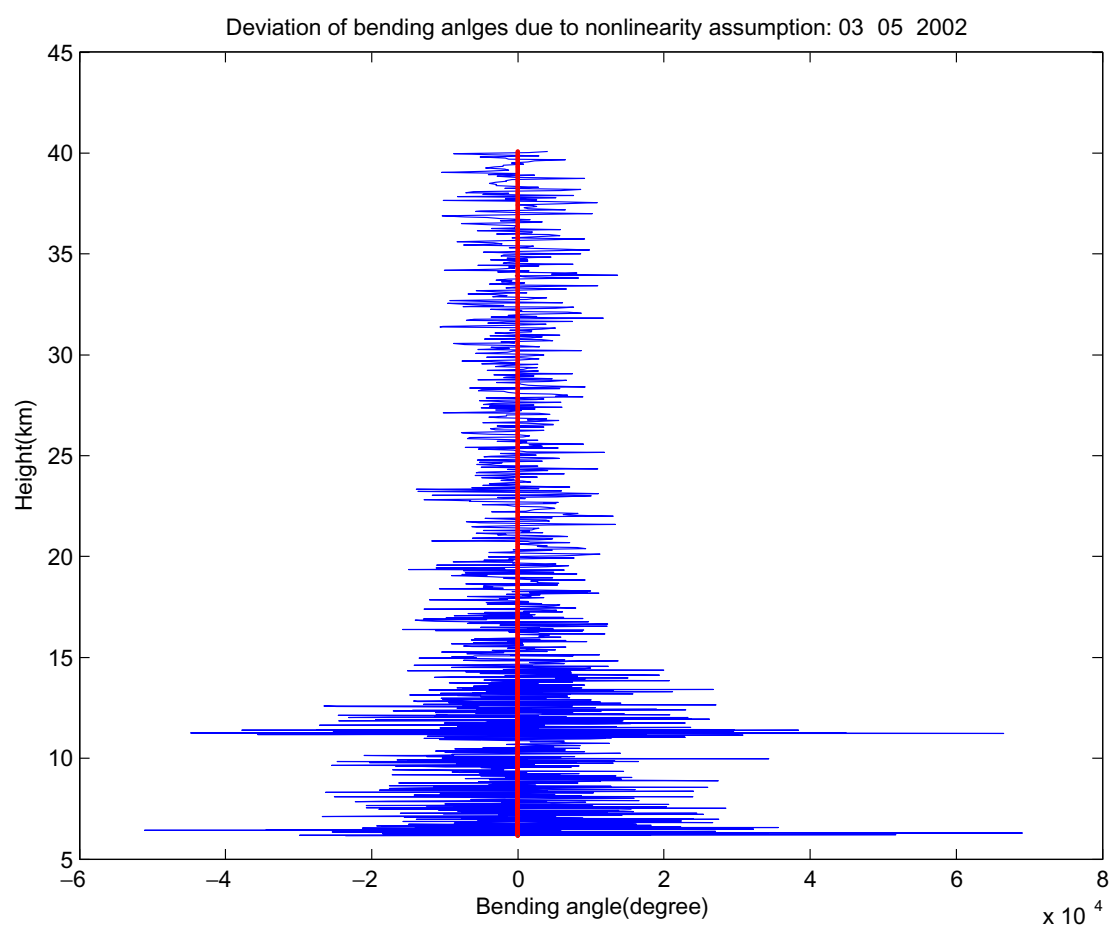

Fig. 14. Differences of computed bending angles due to nonlinearity for occultation 133 of 3rd May 2002.

$\alpha(a)_{i}$ obtained are plotted in Fig. 14 which indicates the nonlinearity error to increase with decreasing atmospheric height. From $40 \mathrm{~km}$ to $15 \mathrm{~km}$, the deviation is within $\pm 2 \times 10^{-4}$ (degrees) but increases to $\pm 7 \times 10^{-4}$ (degrees) for the region below $15 \mathrm{~km}$ with the maximum absolute deviation of $0.00069^{\circ}$ for this particular example. This maximum absolute error is less than $1 \%$. Vorob'ev and Krasil'nikova (1994) pointed out that the error due to nonlinearity increases downwards to a maximum of about $2 \%$ when the beam perigee is close to the Earth's ground. The large difference in computed bending angles with decrease in height is expected as the region below $5 \mathrm{~km}$ is affected by the presence of water vapour and as seen from Fig. 7, the bending angles due to L2 are highly nonlinear.

The analytical approach was next tested by being used to compute the bending angles of occultation number 3 of 14th May 2001 which occurred past mid-night at 00:39:58.00. For this period as stated earlier, the solar radiation is min- 


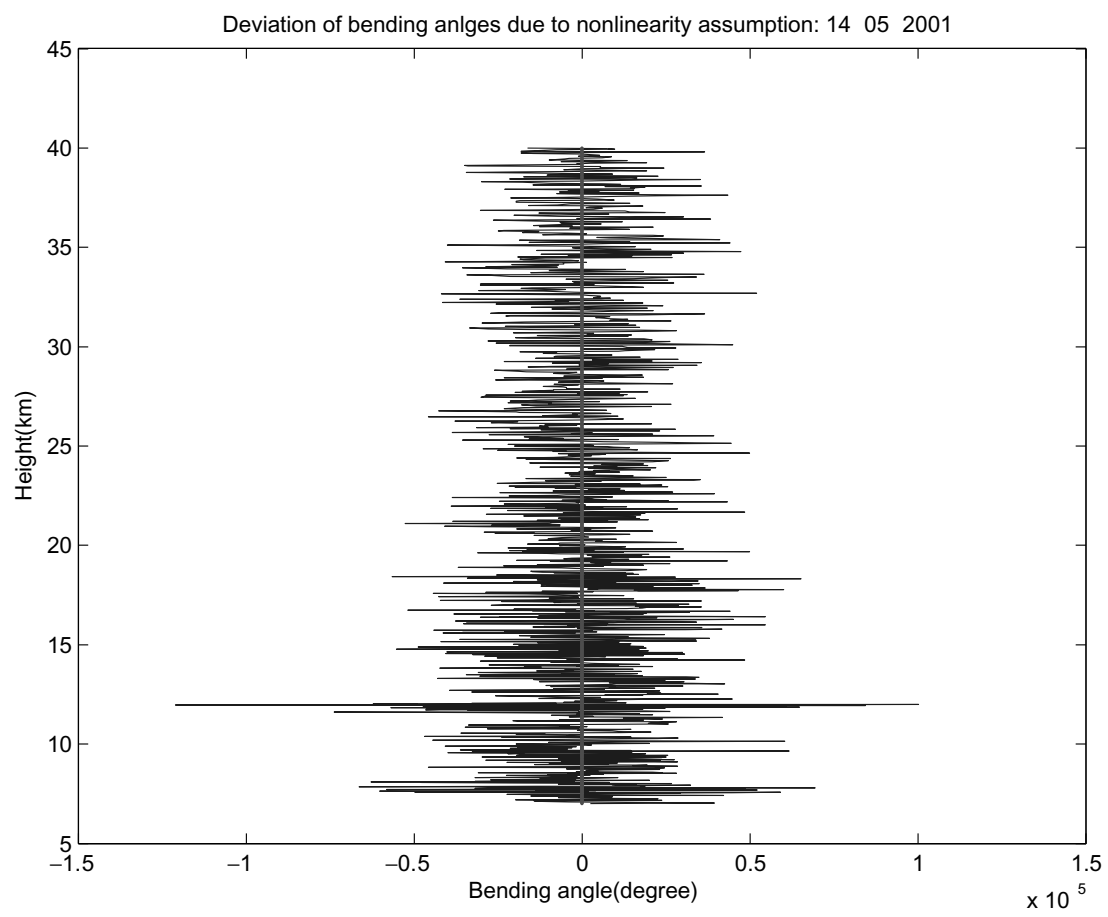

Fig. 15. Differences of computed bending angles due to nonlinearity for occultation number 3 of 14 th May 2001 .

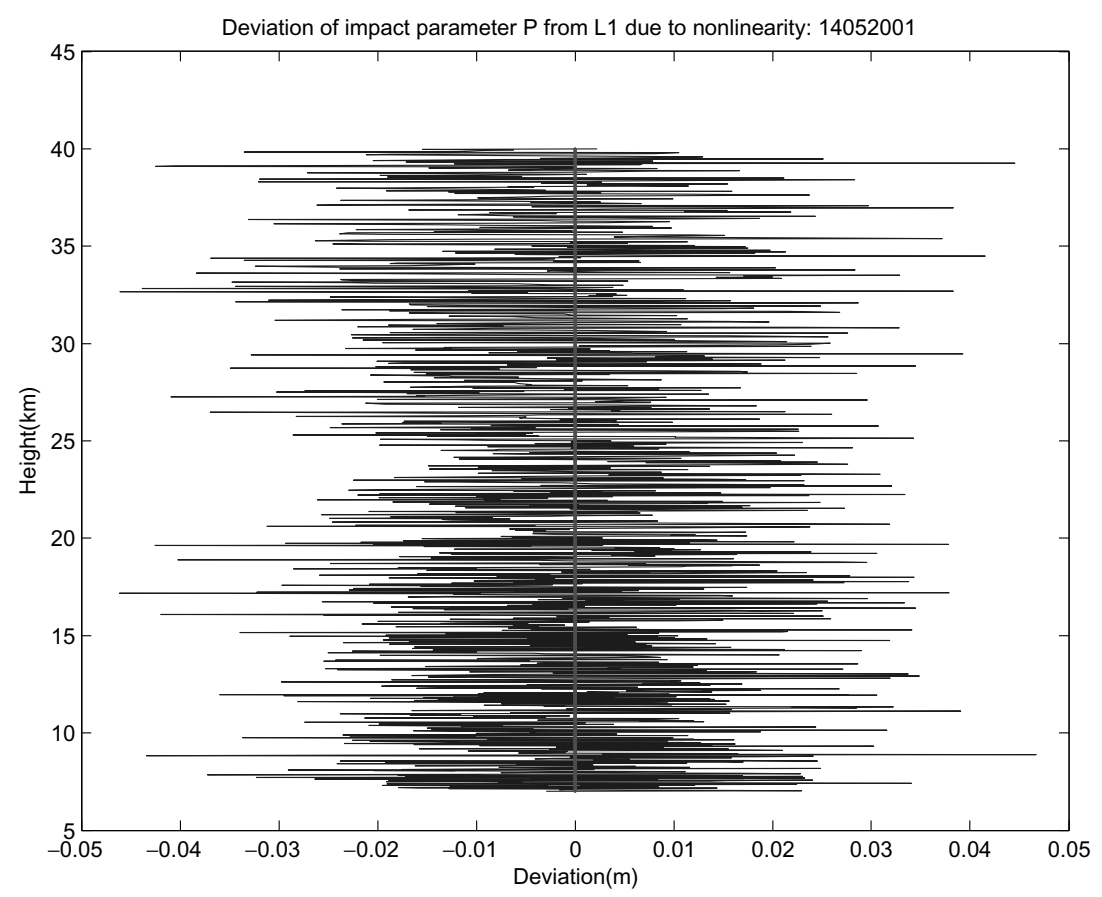

Fig. 16. Differences in computed impact parameters from L1 due to nonlinearity for occultation number 3 of 14th May 2001.

imum and the effect of ionospheric noise is also minimum. Further, as an additional test, the analytical algorithms were used to compute the bending angles of occultation number 6 of 2nd February 2002 which occurred shortly passed midnight. The results from occultation number 3 of 14th May 2001 show the differences in bending angles from the analytical and Newton's methods to be smaller (see Fig. 15). The maximum absolute difference value for bending angles was $0.00001^{\circ}$. For the computed impact parameters, the differences were in the range $\pm 5 \mathrm{~cm}$ for L1 signal (Fig. 16) and $\pm 6 \mathrm{~cm}$ for L2 (Fig. 17). The maximum absolute values were $4 \mathrm{~cm}$ and $5 \mathrm{~cm}$ respectively. In comparison to the results of occultation 133 of 3rd May 2002, the results of occultation 3 of 14th May 2001 indicate the effect of ionospheric noise during the period of low solar radiation to be less. The ionospheric noise could therefore increase the errors due to nonlinearity. Results from occultation number 6 of 2nd February 2002, which occurred during the period of low solar radiation, however showed increased differences in computed bending angles and impact parameters (Figs. 18, 


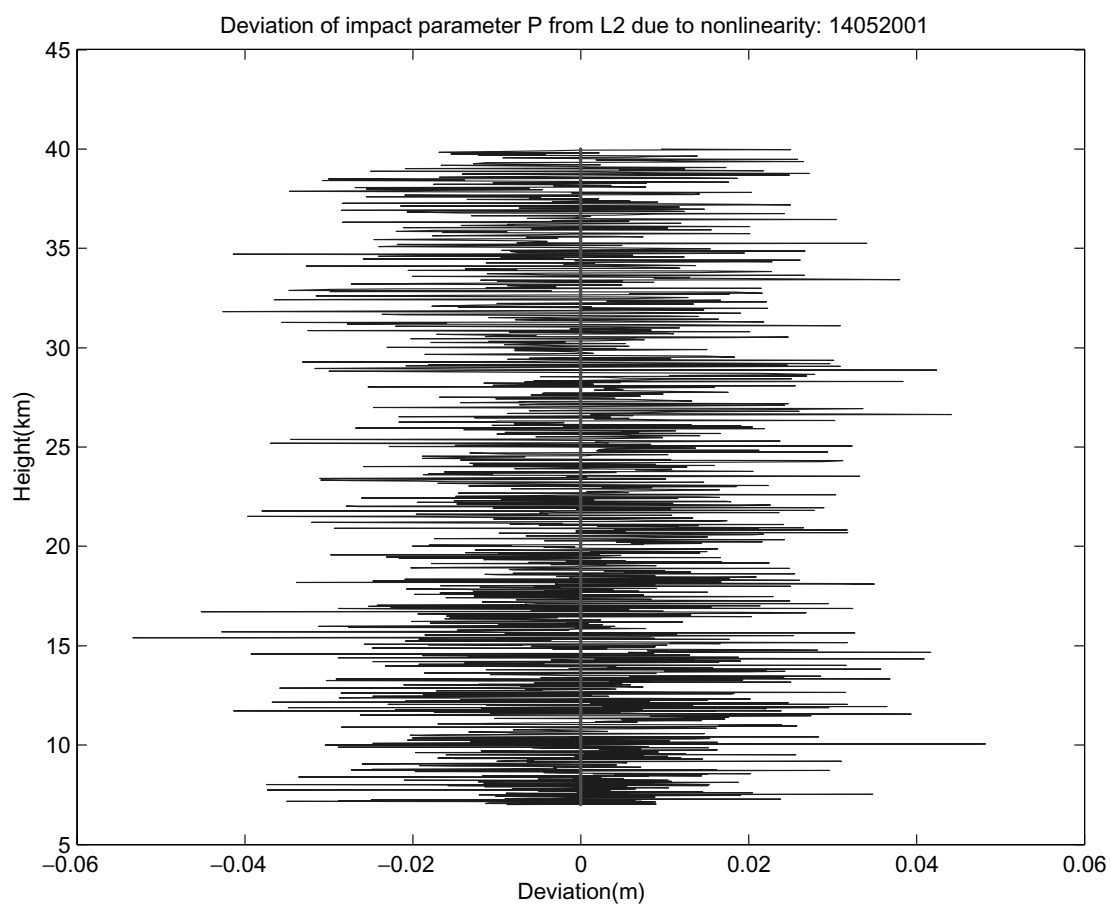

Fig. 17. Differences in computed impact parameters from L2 due to nonlinearity for occultation number 3 of 14th May 2001.

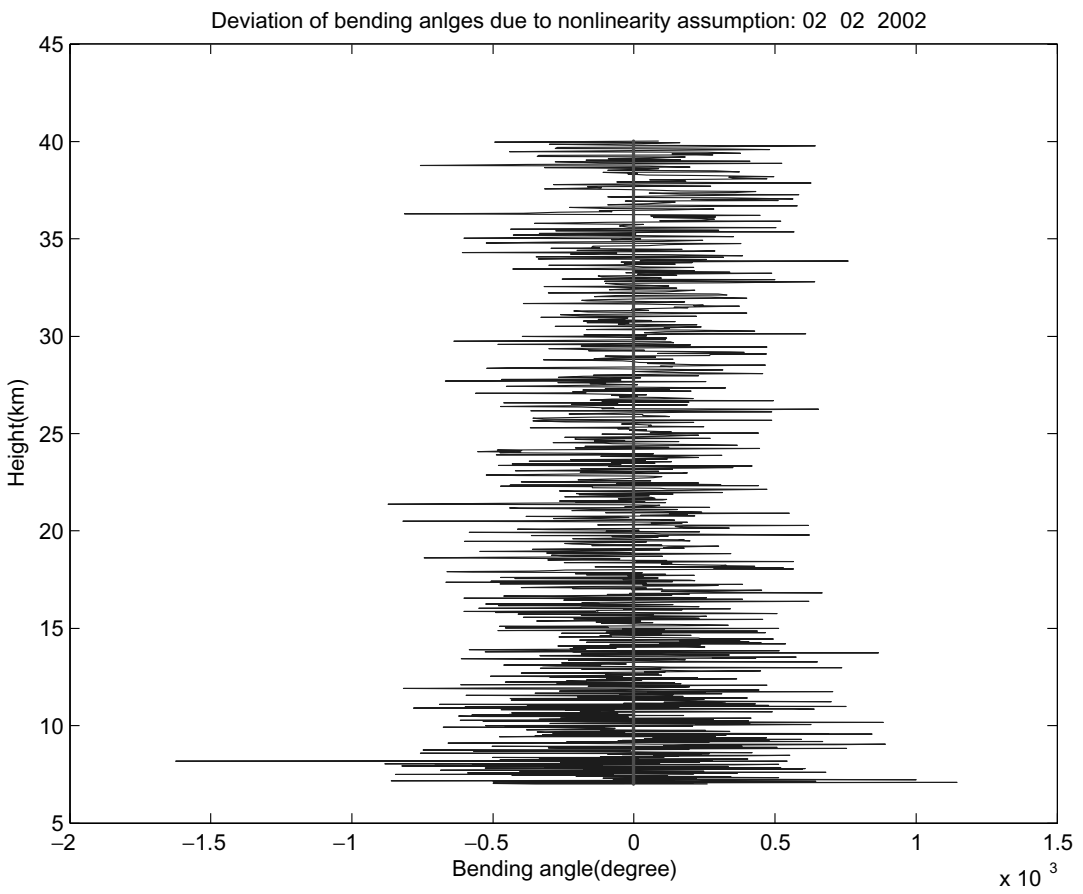

Fig. 18. Differences of computed bending angles due to nonlinearity for occultation number 6 of 2nd February 2002.

19 and 20). There could exist other factors that influence the nonlinearity error other than the ionospheric noise which require further investigations.

\section{Conclusion}

The study has successfully presented an independent analytic algorithm for solving the system of nonlinear bending angles for GPS atmospheric sounding and shown that nonlinearity correction should be taken into account if the accuracy of the desired profiles are to be achieved to $1 \%$ accuracy. In particular, it has been highlighted how the nonlinearity errors in bending angles contribute to errors in the impact parameters to which the bending angles are related. Occultation number 133 of 3rd May 2002 which occurred past noon and occultation number 3 of 14th May 2001 which occurred past mid-night indicated the significance of ionospheric noise on the nonlinearity error. When ionospheric noise is minimum e.g. at mid-night, the computed differences in bending angles between the two procedures are almost negligible. During maximum solar radiation in the afternoons with increased 


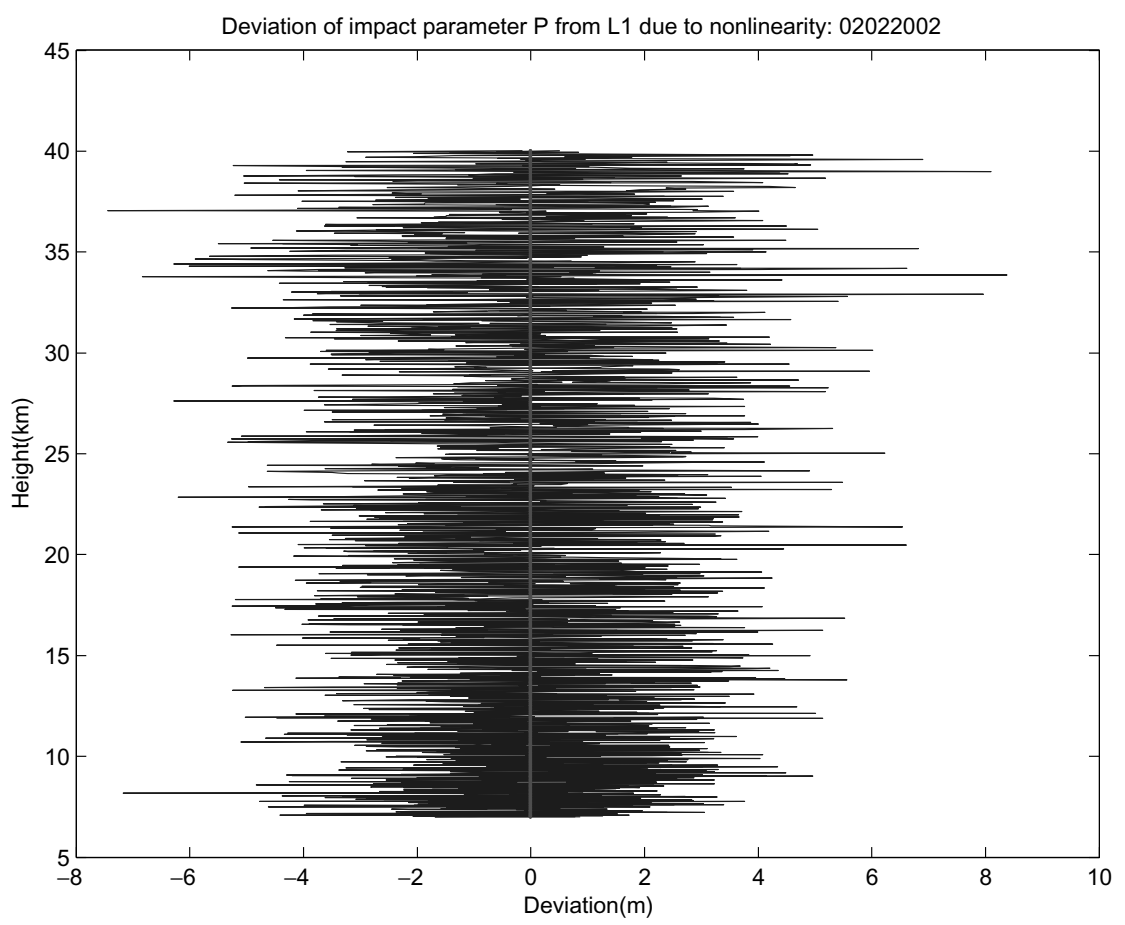

Fig. 19. Differences in computed impact parameters from L1 due to nonlinearity for occultation number 6 of 2nd February 2002.

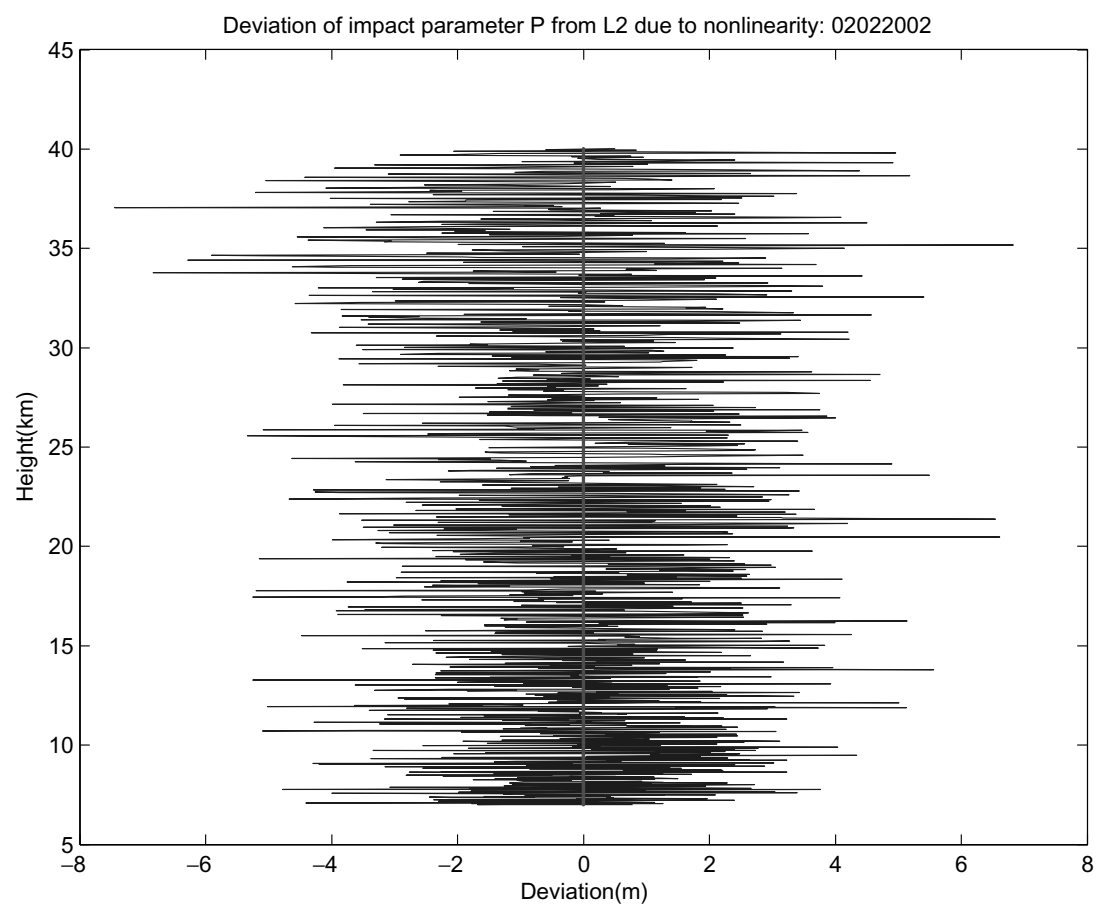

Fig. 20. Differences in computed impact parameters from L2 due to nonlinearity for occultation number 6 of 2nd February 2002.

ionospheric noise, the computed differences in bending angles between the analytical and classical Newton's methods increases. The proposed analytical method could therefore be used to control the results of the classical Newton's method especially when the ionospheric noise is suspected to be great as for occultations that occur during maximum solar radiation periods. The hurdle that must be overcome however is to concretely identify the criteria for selecting the correct solution amongst the four analytical solutions. In this study, the smallest values amongst the four analytic solutions turned out to be the correct ones compared with values of the classical Newton's approach. Whether this applies in general is still subject to investigation. The causes of the large differences in the results of occultation number 6 of 2nd February 2002 during minimum solar radiation period is subject to further investigations. In terms of computing time, the analytical approach would probably have an advantage over the classical Newton's iterative procedure in cases where thou- 
sands of occultations are to be processed. For single occultations however, the classical Newton's approach generally converges after few iterations and as such, the advantage of the analytical approach in light of modern computers may not be so significant.

The proposed analytical procedure has the following advantages:

- The procedure offers a direct solution to the nonlinear equations for bending angles. Errors due to nonlinearity in general could be minimized.

- With this technique, linearization problems and iterative procedures of computation could be avoided.

- Significant computation time could be saved.

- The analytical approach could be used to control classical procedures.

Acknowledgments. The first author wishes to acknowledge the support of JSPS (Japan Society of Promotion of Science) for the financial support that enabled the undertaking of this study at the Department of Geophysics, Kyoto University, Japan. The author is further grateful for the support and the good working atmosphere provided by his host Professors S. Takemoto and Y. Fukuda of the Department of Geophysics, Graduate School of Science, Kyoto University - Japan. Special thanks to Dr. A. Steiner of the Institute of Meteorology and Geophysics, University of Graz, Austria for the support in terms of literature and discussions. The data used in this study were provided by GeoForschungsZentrum Potsdam (GFZ). For these, the authors express their utmost appreciation.

\section{Appendices}

\section{Appendix A: Coefficients of fourth order polynomial} (Equation (5))

$$
\begin{aligned}
& d_{1}=b_{4}^{2} \\
& d_{2}=2 b_{4} b_{5} \\
& d_{3}= 2 b_{4} b_{3} \\
& d_{4}= 2 b_{6} b_{4}+b_{5}^{2}+b_{7}^{2} \\
& d_{5}= 2 b_{1} b_{4}+b_{3}^{2}-b_{7}^{2} \\
& d_{6}= 2 b_{3} b_{5}+2 b_{2} b_{4} \\
& d_{7}= 2 b_{6} b_{5} \\
& d_{8}= 2 b_{1} b_{3} \\
& d_{9}= 2 b_{1} b_{5}+2 b_{2} b_{3} \\
& d_{10}= 2 b_{3} b_{6}+2 b_{5} b_{2} \\
& d_{11}= b_{1}^{2} \\
& d_{12}= 2 b_{1} b_{2} \\
& d_{13}= 2 b_{1} b_{6}+b_{2}^{2}+b_{7}^{2} \\
& d_{14}= 2 b_{2} b_{6} \\
& d_{15}= b_{6}^{2}-b_{7}^{2} \\
& w_{i t h} \\
& b_{1}= a_{1}^{2}+a_{2}^{2} \\
& b_{2}=-2 a a_{2} \\
& b_{3}= 2 a_{2} a_{4} \\
& b_{4}=\left(a_{3}^{2}+a_{4}^{2}\right) \\
& b_{5}=-2 a a_{4} \\
& b_{6}=a^{2}-a_{1}^{2}-a_{3}^{2} \\
& b_{7}=2 a_{1} a_{3}
\end{aligned}
$$

\section{Appendix B: Coefficients of quartic polynomials (Equa-} tion (18))

$$
\begin{aligned}
& g_{4}=\left(a_{6}^{4} d_{1}+a_{5}^{4} d_{11}-a_{5} a_{6}^{3} d_{3}+a_{5}^{2} a_{6}^{2} d_{5}-a_{5}^{3} a_{6} d_{8}\right) \\
& g_{3}=\left(a_{5}^{4} d_{12}-a_{5} a_{6}^{3} d_{2}+a_{5}^{2} a_{6}^{2} d_{6}-a_{5}^{3} a_{6} d_{9}\right) \\
& g_{2}=\left(-a_{5}^{3} a_{6} d_{10}+a_{5}^{4} d_{13}+a_{5}^{2} a_{6}^{2} d_{4}\right) \\
& g_{1}=\left(a_{5}^{4} d_{14}-a_{5}^{3} a_{6} d_{7}\right) ; \\
& g_{0}=a_{5}^{4} d_{15}
\end{aligned}
$$

and

$$
\begin{aligned}
& h_{4}=\left(a_{6}^{4} d_{1}+a_{5}^{4} d_{11}-a_{5} a_{6}^{3} d_{3}+a_{5}^{2} a_{6}^{2} d_{5}-a_{5}^{3} a_{6} d_{8}\right) \\
& h_{3}=\left(-a_{5}^{3} a_{6} d_{12}+a_{6}^{4} d_{2}-a_{5} a_{6}^{3} d_{6}+a_{5}^{2} a_{6}^{2} d_{9}\right) \\
& h_{2}=\left(-a_{5} a_{6}^{3} d_{10}+a_{5}^{2} a_{6}^{2} d_{13}+a_{6}^{4} d_{4}\right) \\
& h_{1}=\left(-a_{5} a_{6}^{3} d_{14}+a_{6}^{4} d_{7}\right) \\
& h_{0}=a_{6}^{4} d_{1} 5
\end{aligned}
$$

\section{References}

Awange, J. L., Groebner basis solution of planar resection, Survey Review, 36, 528-543, 2002.

Awange, J. L. and E. Grafarend, Sylvester resultant solution of planar ranging problem, Allgemeine Vermessungs-Nachrichten, 108, 143-146, 2002.

Awange, J. L. and E. Grafarend, Groebner basis solution of the threedimensional resection problem (P4P), Journal of Geodesy, 77, 327-337, 2003a.

Awange, J. L. and E. Grafarend, Explicit solution of the overdetermined three-dimension resection problem, Journal of Geodesy, 76, 605-616, 2003 b.

Awange, J. L., E. Grafarend, Y. Fukuda, and S. Takemoto, Direct Polynomial approach to nonlinear distance (ranging) problems, Earth Planets Space, 55, 231-241, 2003.

Becker, T. and V. Weispfenning, Groebner bases. A computational approach to commutative algebra. Graduate Text in Mathematics 141, 2nd Edition, Springer-Verlag, New York, 1998.

Buchberger, B., Ein Algorithmisches Kriterium für die Lösbarkeit eines algebraischen Gleichungsystems, Aequationes Mathematicae, 4, 374383,1970

Buchberger, B., Groebner bases. A short introduction for system theorists, in EUROCAST 2001, LNCS 2178, edited by R. Moreno-Diaz et al., 1-19, 2001.

Cox, D., J. Little and D. O'Shea, Using algebraic geometry. Graduate Text in Mathematics 185, 499 pp., Springer-Verlag, New York, 1998.

Gurbunov, M. E., A. S. Gurvich, and L. Bengtsson, Advanced algorithms of inversion of GPS/MET satellite data and their application to the reconstruction of temperature and humidity, Rep. No. 211, Max-Plunk-Institut für Meteorologie, Hamburg, Germany, 1996.

Kursinski, E. R., G. A. Hajj, J. T. Schofield, R. P. Linfield, and K. R. Hardy, Observing Earth's atmosphere with radio occultation measurements using the Global Positioning System, J. Geophys. Res., 102, 23,429-23,465, 1997.

Healey, S., A. Jupp, D. Offiler, and J. Eyre, The assimilation of radio occultation measurements, in First CHAMP Mission Results for Gravity, Magnetic and Atmospheric Studies edited by C. Reigber, H. Lhr, and P. Schwintzer, Springer-Verlag, Heidelberg, 453-461, 2003.

Steiner, A. K., High resolution sounding of key climate variables using the radio occultation technique, Dissertation, Institute for Meteorology and Geophysics, University of Graz, No. 3, 1998.

Sturmfels, B., Introduction to resultants, AMS Proceedings of Symposia in Applied Mathematics, 53, 25-39, 1998.

Vorob'ev, V. V. and T. G. Krasil'nikova, Estimation of the accuracy of atmospheric refractive index recovery from Doppler shift measurements at frequencies used in the NAVSTAR system, Phys. of Atmos. and Oceans, 29, 602-609, 1994.

Wickert, J., Das CHAMP-Radiookkultationsexperiment: Algorithmen, Prozessierungssystem und Ergebnisse, Scientific Technical Report STR02/07, GFZ, Potsdam, 2002.

J. L. Awange (e-mail: awange@kugi.kyoto-u.ac.jp), Y. Fukuda, S. Takemoto, J. Wickert, and Y. Aoyama 